\title{
A Toeplitz-Like Operator with Rational Symbol Having Poles on the Unit Circle III: The Adjoint
}

\author{
G. J. Groenewald, S. ter Horst, J. Jaftha and A. C. M. Ran
}

\begin{abstract}
This paper contains a further analysis of the Toeplitz-like operators $T_{\omega}$ on $H^{p}$ with rational symbol $\omega$ having poles on the unit circle that were previously studied in Groenewald (Oper Theory Adv Appl 271:239-268, 2018; Oper Theory Adv Appl 272:133-154, 2019). Here the adjoint operator $T_{\omega}^{*}$ is described. In the case where $p=2$ and $\omega$ has poles only on the unit circle $\mathbb{T}$, a description is given for when $T_{\omega}^{*}$ is symmetric and when $T_{\omega}^{*}$ admits a selfadjoint extension. If in addition $\omega$ is proper, it is shown that $T_{\omega}^{*}$ coincides with the unbounded Toeplitz operator defined by Sarason (Integr Equ Oper Theory 61:281298, 2008) and studied further by Rosenfeld (Classes of densely defined multiplication and Toeplitz operators with applications to extensions of RKHS's, 2013; J Math Anal Appl 440:911-921, 2016).
\end{abstract}

Mathematics Subject Classification. Primary 47B35, 47A53; Secondary 47A68.

Keywords. Toeplitz operators, Unbounded operators, Adjoint, Symmetric operators.

\section{Introduction}

In this paper we proceed with our study of unbounded Toeplitz-like operators on $H^{p}$ with rational symbols that have poles on the unit circle $\mathbb{T}$ which was initiated in [4]. Our previous work on such Toeplitz-like operators focused on their Fredholm properties (in [4]) and the various parts of their spectra (in [5]). Here we determine properties of the adjoint operator and conditions

This work is based on the research supported in part by the National Research Foundation of South Africa (Grant Nos. 90670 and 118513). Part of the research was done during a visit by the third author to VU Amsterdam supported through the Teaching Development Grant National Collaborative Project: Strengthening Academic Staff Development in Mathematical and Statistical Sciences in South Africa (Grant Nos. APP-TDG-135 and APP-TDG-136). 
under which the operator is symmetric and when it has a selfadjoint extension.

Before we can define our Toeplitz-like operators, some notation has to be introduced. We write Rat for the space of rational complex functions, $\operatorname{Rat}(\mathbb{T})$ for the subspace of Rat consisting of rational complex functions with poles only on the unit circle $\mathbb{T}$, and $\operatorname{Rat}_{0}(\mathbb{T})$ for the subspace of strictly proper functions in $\operatorname{Rat}(\mathbb{T})$. Now let $\omega \in$ Rat, possibly with poles on $\mathbb{T}$. As in [4], we define the Toeplitz-like operator $T_{\omega}\left(H^{p} \rightarrow H^{p}\right)$, for $1<p<\infty$, via

$$
\operatorname{Dom}\left(T_{\omega}\right)=\left\{g \in H^{p} \mid \omega g=f+\rho \text { with } f \in L^{p}, \rho \in \operatorname{Rat}_{0}(\mathbb{T})\right\}, T_{\omega} g=\mathbb{P} f .
$$

Here $\mathbb{P}$ is the Riesz projection of $L^{p}$ onto $H^{p}$. The operator $T_{\omega}$ is densely defined and closed. In case $\omega \in \operatorname{Rat}(\mathbb{T})$, explicit formulas for the domain, kernel, range, and a complement of the range were obtained in [5], as an extension of a result in [4] for the case where $T_{\omega}$ is Fredholm. We recall these results in Sect. 2, as they will be frequently used throughout the paper.

If $\omega$ has no poles on $\mathbb{T}$, in fact for any $\omega \in L^{\infty}$, the adjoint of the Toeplitz operator $T_{\omega}$ on $H^{p}$ can be identified with the Toeplitz operator $T_{\omega^{*}}$ on $H^{p^{\prime}}$, with $1<p^{\prime}<\infty$ so that $1 / p+1 / p^{\prime}=1$ and with $\omega^{*}$ defined as $\omega^{*}(z)=\overline{\omega(z)}$ on $\mathbb{T}$. The identification of $\left(H^{p}\right)^{\prime}$ and $H^{p^{\prime}}$ goes via the usual pairing

$$
\langle f, g\rangle_{p, p^{\prime}}=\frac{1}{2 \pi} \int_{\mathbb{T}} \overline{g(z)} f(z) d z \quad\left(f \in H^{p}, g \in H^{p^{\prime}}\right) .
$$

In the sequel we use the same notation for the similarly defined pairing between $L^{p}$ and $L^{p^{\prime}}$ to identify $\left(L^{p}\right)^{\prime}$ and $L^{p^{\prime}}$, and in both cases the indices will often be omitted.

For the Toeplitz-like operators studied in this paper the situation is more complicated than for Toeplitz operators with $L^{\infty}$ symbols. However, we do obtain that $T_{\omega}^{*}$ can be identified with the restriction of the Toeplitzlike operator $T_{\omega^{*}}$ on $H^{p^{\prime}}$ to a dense subspace of its domain. Like for the operator $T_{\omega}$, in case $\omega$ is in $\operatorname{Rat}(\mathbb{T})$ we obtain a more explicit description of $T_{\omega}^{*}$, which we present after introducing some further notation.

Throughout the paper $\mathcal{P}$ denotes the space of complex polynomials and $\mathcal{P}_{k}$, for any non-negative integer $k$, denotes the subspace of $\mathcal{P}$ of polynomials of degree at most $k$. The degree of a polynomial $r \in \mathcal{P}$ is denoted as $\operatorname{deg}(r)$. Given $r \in \mathcal{P}$ with $\operatorname{deg}(r)=k$, say $r(z)=r_{0}+z r_{1}+\cdots+z^{k} r_{k}$, we define the polynomial $r^{\sharp}$ by

$$
r^{\sharp}(z)=z^{k} \overline{r(1 / \bar{z})}=\overline{r_{0}} z^{k}+\overline{r_{1}} z^{k-1}+\cdots+\overline{r_{k}} .
$$

The following theorem is our first main result.

Theorem 1.1. Let $\omega=s / q \in$ Rat with $s, q \in \mathcal{P}$ co-prime and $1<p<\infty$. Factor $s=s_{-} s_{0} s_{+}$and $q=q_{-} q_{0} q_{+}$with $s_{-}, q_{-}$having roots only inside $\mathbb{T}$, $s_{0}, q_{0}$ having roots only on $\mathbb{T}$, and $s_{+}, q_{+}$having roots only outside $\mathbb{T}$. Set $m=\operatorname{deg}(q), n=\operatorname{deg}(s), m_{ \pm}=\operatorname{deg}\left(q_{ \pm}\right), n_{ \pm}=\operatorname{deg}\left(s_{ \pm}\right) m_{0}=\operatorname{deg}\left(q_{0}\right)$, $n_{0}=\operatorname{deg}\left(s_{0}\right)$ and let $1<p^{\prime}<\infty$ with $1 / p+1 / p^{\prime}=1$. Then

$$
\operatorname{Dom}\left(T_{\omega}^{*}\right)=\left(q_{0}\right)^{\sharp} H^{p^{\prime}} \subset \operatorname{Dom}\left(T_{\omega^{*}}\right) \quad \text { and } \quad T_{\omega}^{*}=\left.T_{\omega^{*}}\right|_{\left(q_{0}\right)^{\sharp} H^{p^{\prime}}} \text {. }
$$


Furthermore, we have

$$
\begin{aligned}
& \operatorname{Ran}\left(T_{\omega}^{*}\right)=T_{z^{m-n}\left(s_{+}\right)^{\sharp} /\left(q_{+}\right)^{\sharp}} Q_{n_{0}+n_{-}-m_{0}-m_{-}}\left(s_{0}\right)^{\sharp} H^{p^{\prime}}, \\
& \operatorname{Ker}\left(T_{\omega}^{*}\right)=\left\{\frac{\left(q_{-}\right)^{\sharp}\left(q_{0}\right)^{\sharp} r}{\left(s_{-}\right)^{\sharp}} \mid \operatorname{deg}(r)<n_{-}-m_{-}-m_{0}\right\} .
\end{aligned}
$$

Here $Q_{k}=I_{H^{p^{\prime}}}-P_{\mathcal{P}_{k-1}}$, with $P_{\mathcal{P}_{k-1}}$ the standard projection in $H^{p^{\prime}}$ onto $\mathcal{P}_{k-1} \subset H^{p^{\prime}}$ to be interpreted as 0 if $k \leq 0$, i.e., $Q_{k}=I_{H^{p^{\prime}}}$ if $k \leq 0$. Thus, for $n_{0}+n_{-} \leq m_{0}+m_{-}$we have $\operatorname{Ran}\left(T_{\omega}^{*}\right)=T_{z^{m-n}} /\left(q_{+}\right)^{\sharp}\left(s_{+} s_{0}\right)^{\sharp} H^{p^{\prime}}$. Moreover,

$\operatorname{dim} \operatorname{Ker}\left(T_{\omega}^{*}\right)=\max \{0, \#\{$ zeroes of $\omega$ inside $\mathbb{D}\}-\#\{$ poles of $\omega$ in $\overline{\mathbb{D}}\}\}$, where the multiplicities of the zeroes and poles are taken into account. Hence, $\operatorname{dim} \operatorname{Ker}\left(T_{\omega}^{*}\right)$ is the maximum of 0 and $n_{-}-m_{-}-m_{0}$. In particular, $T_{\omega}^{*}$ is injective if and only if the number of poles of $\omega$ inside $\overline{\mathbb{D}}$ is greater than or equal to the number of zeroes of $\omega$ inside $\mathbb{D}$, multiplicities taken into account.

Before giving a proof of Theorem 1.1 in Sect. 4, we prove the specialization of this result for the case $\omega \in \operatorname{Rat}(\mathbb{T})$ in Sect. 3. For this purpose we first provide a description of $T_{\omega^{*}}$ in Sect. 2.

The injectivity result, but not the description of $\operatorname{Ker}\left(T_{\omega}^{*}\right)$, can also be derived from general theory and results on $T_{\omega}$. Indeed, according to Theorem II.3.7 in [3], $T_{\omega}^{*}$ is injective if and only if $T_{\omega}$ has dense range, so that the claim follows from Proposition 2.4 in [5]. More can be obtained in this way, since $H^{p}, 1<p<\infty$, is reflexive. By Theorem II.2.14 of [3] it follows that $T_{\omega}^{* *}=T_{\omega}$, with the usual identifications of the dual spaces. Hence, applying the above to $T_{\omega}^{*}$ we find that $T_{\omega}^{*}$ has dense range if and only if $T_{\omega}$ is injective; see also Theorem II.4.10 in [3]. By Banach's Closed Range Theorem, cf., [14], $T_{\omega}^{*}$ has closed range if and only if $T_{\omega}$ has closed range. Again applying results from [5] now gives the following result.

Corollary 1.2. Let $\omega \in$ Rat and $1<p<\infty$. Then $T_{\omega}^{*}$ has closed range if and only if $\omega$ has no zeroes on $\mathbb{T}$, or equivalently, $\omega^{*}$ has no zeroes on $\mathbb{T}$. Moreover, $T_{\omega}^{*}$ has dense range if and only if

$$
\#\left\{\begin{array}{l}
\text { poles of } \omega \text { inside } \overline{\mathbb{D}} \\
\text { multi. taken into account }
\end{array}\right\} \leq \#\left\{\begin{array}{l}
\text { zeroes of } \omega \text { inside } \overline{\mathbb{D}} \\
\text { multi. taken into account }
\end{array}\right\} \text {. }
$$

Beyond Sect. 4, and in the remainder of this introduction, we only consider the case $p=2$ and $\omega \in \operatorname{Rat}(\mathbb{T})$. By comparing the results on $T_{\omega}$ and $T_{\omega}^{*}$ it is obvious $T_{\omega}$ cannot be selfadjoint, except when $\omega$ has no poles on $\mathbb{T}$. In Sect. 5 we describe in terms of $\omega$ when $T_{\omega}^{*}$ is symmetric, in which case $T_{\omega}^{*} \subset T_{\omega}$, and whenever $T_{\omega}^{*}$ is symmetric we describe when $T_{\omega^{*}}$ admits a selfadjoint extension. The following theorem collects some of the main results of Sect. 5; it follows directly from Theorem 5.1, Corollaries 5.2 and 5.7, Propositions 5.4 and 5.9.

Theorem 1.3. Let $\omega=s / q \in \operatorname{Rat}(\mathbb{T})$ with $s, q \in \mathcal{P}$ co-prime. Consider $T_{\omega}$ on $H^{2}$. Then

$$
T_{\omega}^{*} \text { is symmetric } \Longleftrightarrow \omega(\mathbb{T}) \subset \mathbb{R} \text {. }
$$


In particular, if $T_{\omega}^{*}$ is symmetric, then $\operatorname{deg}(s) \leq \operatorname{deg}(q) \leq 2 \operatorname{deg}(s)$. Furthermore, if $T_{\omega}^{*}$ is symmetric, then $T_{\omega}^{*}$ admits a selfadjoint extension if and only if the number of roots of $s-i q$ and $s+i q$ in $\mathbb{D}$, counting multiplicities, coincide. This happens in particular if $\omega(\mathbb{T}) \neq \mathbb{R}$, but cannot happen in case $\operatorname{deg}(q)$ is odd.

Several other conditions for $T_{\omega}^{*}$ to be symmetric and/or have a selfadjoint extension are derived in Sect. 5 .

In [11] Sarason introduced and studied an unbounded Toeplitz-like operator with symbol in the Smirnov class. In Sect. 6 we show that if $\omega \in \operatorname{Rat}(\mathbb{T})$ is proper, then the adjoint operator $T_{\omega}^{*}$ is precisely a Toeplitz-like operator of the type studied by Sarason. Hence in this case our Toeplitz-like operator $T_{\omega}=T_{\omega}^{* *}$ coincides with the adjoint of the Toeplitz-like operator considered in [11]. Based on ideas in [11], we also show that $H(\overline{\mathbb{D}})$, the space of functions analytic on a neighborhood of $\overline{\mathbb{D}}$, is contained in $\operatorname{Dom}\left(T_{\omega}\right)$ and in fact is a core of $T_{\omega}$.

In the last section of [11], Sarason introduces a class of closed, densely defined Toeplitz-like operators on $H^{2}$ determined by algebraic properties, which was further investigated by Rosenfeld in $[9,10]$. In particular, this class of Toeplitz-like operators contains the unbounded Toeplitz-like operator studied by Sarason and is closed under taking adjoints, and hence contains our Toeplitz-like operators with proper symbols in $\operatorname{Rat}(\mathbb{T})$. In fact, we will show in Sect. 6 that $T_{\omega}$ is contained in the class of Toeplitz-like operators for any $\omega$ in Rat.

\section{The Operator $T_{\omega *}$ for $\omega \in \operatorname{Rat}(\mathbb{T})$}

In this section we recall some results from $[4,5]$ on the operator $T_{\omega}$ for $\omega \in$ $\operatorname{Rat}(\mathbb{T})$ that we will use in the sequel, and apply them to the operator $T_{\omega^{*}}$. Hence, throughout this section let $\omega=s / q \in \operatorname{Rat}(\mathbb{T})$, with $s, q \in \mathcal{P}$ co-prime. We set $m=\operatorname{deg}(q)$ and $n=\operatorname{deg}(s)$. Furthermore, factor $s=s_{-} s_{0} s_{+}$with $s_{-}$, $s_{0}$ and $s_{+}$polynomials having roots only inside, on, or outside $\mathbb{T}$, respectively. We then recall from Theorem 2.2 in [5] that

$$
\begin{aligned}
\operatorname{Ker}\left(T_{\omega}\right) & =\left\{r / s_{+} \mid \operatorname{deg}(r)<m-\operatorname{deg}\left(s_{-} s_{0}\right)\right\} ; \\
\operatorname{Dom}\left(T_{\omega}\right) & =q H^{p}+\mathcal{P}_{m-1} ; \quad \operatorname{Ran}\left(T_{\omega}\right)=s H^{p}+\widetilde{\mathcal{P}},
\end{aligned}
$$

where $\widetilde{\mathcal{P}}$ is the subspace of $\mathcal{P}$ given by

$$
\widetilde{\mathcal{P}}=\left\{r \in \mathcal{P} \mid r q=r_{1} s+r_{2} \text { for } r_{1}, r_{2} \in \mathcal{P}_{m-1}\right\} \subset \mathcal{P}_{n-1} .
$$

Furthermore, $H^{p}=\overline{\operatorname{Ran}\left(T_{\omega}\right)}+\widetilde{\mathcal{Q}}$ forms a direct sum decomposition of $H^{p}$, where

$$
\widetilde{\mathcal{Q}}=\mathcal{P}_{k-1} \quad \text { with } \quad k=\max \left\{\operatorname{deg}\left(s_{-}\right)-m, 0\right\},
$$

using the convention $\mathcal{P}_{-1}:=\{0\}$. Furthermore, the action of $T_{\omega}$ is as follows:

$$
\begin{aligned}
& T_{\omega} g=s h+\widetilde{r} \quad\left(g=q h+r \in q H^{p}+\mathcal{P}_{m-1}=\operatorname{Dom}\left(T_{\omega}\right)\right), \\
& \text { where } \widetilde{r} \in \mathcal{P}_{n-1} \text { is such that } r s=\widetilde{r} q+r_{2} \text { for some } r_{2} \in \mathcal{P}_{m-1} .
\end{aligned}
$$


We also recall from Lemma 5.3 in [4] that

$$
T_{z^{\kappa} \omega}=T_{z^{\kappa}} T_{\omega} \text { for any integer } \kappa \leq 0 .
$$

Recall that $\omega^{*}$ is defined as $\omega^{*}(z)=\overline{\omega(z)}$ on $\mathbb{T}$, i.e., $\omega^{*}(z)=\overline{s(z)} / \overline{q(z)}$. For $z \in \mathbb{T}$

$$
\overline{q(z)}=\overline{q_{0}+z q_{1}+\cdots+z^{m} q_{m}}=\overline{q_{0}}+\overline{q_{1}} \frac{1}{z}+\cdots+\overline{q_{m}} \frac{1}{z^{m}}=\frac{1}{z^{m}} q^{\sharp}(z) .
$$

Hence $q^{\sharp}(z)=z^{m} \overline{q(z)}$, and likewise $s^{\sharp}(z)=z^{n} \overline{s(z)}$. Thus we have

$$
\omega^{*}(z)=\frac{z^{m-n} s^{\sharp}(z)}{q^{\sharp}(z)} \text { if } m \geq n \quad \text { and } \quad \omega^{*}(z)=\frac{s^{\sharp}(z)}{z^{n-m} q^{\sharp}(z)} \text { if } m<n \text {. }
$$

In fact, the formula $\omega^{*}(z)=z^{m-n} s^{\sharp}(z) / q^{\sharp}(z)$ holds in both cases, but is not always a representation as the ratio of two polynomials. Note in particular that $\omega^{*} \in \operatorname{Rat}(\mathbb{T})$ in case $\omega$ is proper, while this need not be the case if $\omega$ is not proper. Thus, if $\omega$ is proper, the above formulas apply directly, while for the non-proper case, using (2.4) we can reduce certain questions to questions concerning the Toeplitz operator $T_{s \sharp} / q^{\sharp}$ with symbol $s^{\sharp} / q^{\sharp}$ which is in $\operatorname{Rat}(\mathbb{T})$.

A polynomial $r \neq 0$ is called self-inversive in case $r=\gamma r^{\sharp}$ for a constant $\gamma \in \mathbb{C}$, which necessarily is unimodular. In fact, $\gamma$ is the ratio $r_{0} / \overline{r_{n}}$ with $r_{0}=r(0)$ and $r_{n}$ the leading coefficient of $r$. By a theorem of Cohn [1], a polynomial $r$ has all its roots on $\mathbb{T}$ if and only if $r$ is self-inversive and its derivative has all its roots in the closed unit disc $\overline{\mathbb{D}}$. Hence, any polynomial with roots only on $\mathbb{T}$ is self-inversive. In particular, $q=\gamma q^{\sharp}$ and $s_{0}=\rho\left(s_{0}\right)^{\sharp}$ for unimodular constants $\gamma$ and $\rho$.

More generally, in the transformation $r \rightarrow r^{\sharp}$, the nonzero roots of $r$ (including multiplicity) transfer along the unit circle via the map $\alpha \mapsto 1 / \bar{\alpha}=$ $|\alpha|^{-2} \alpha$, while the degree decreases by the multiplicity of 0 as a root of $r$. Consequently, in the factorization $s^{\sharp}=\left(s_{+}\right)^{\sharp}\left(s_{0}\right)^{\sharp}\left(s_{-}\right)^{\sharp}$, the polynomials $\left(s_{+}\right)^{\sharp}$, $\left(s_{0}\right)^{\sharp}$ and $\left(s_{-}\right)^{\sharp}$ contain the roots of $s^{\sharp}$ inside, on and outside $\mathbb{T}$, respectively, taking multiplicities into account. We write $\left(s_{+}\right)^{\sharp}$ rather than $s_{+}^{\sharp}$, etc., to avoid confusion with what one may interpret as $\left(s^{\sharp}\right)_{+}$.

We now apply the above to $T_{\omega^{*}}$ acting on $H^{p^{\prime}}, 1<p^{\prime}<\infty$, to fit better with the remainder of the paper.

Proposition 2.1. Let $\omega=s / q \in \operatorname{Rat}(\mathbb{T})$, with $s, q \in \mathcal{P}$ co-prime, $m=\operatorname{deg}(q)$ and $n=\operatorname{deg}(s)$. Factor $s=s_{-} s_{0} s_{+}$with $s_{-}, s_{0}$ and $s_{+}$polynomials having roots only inside, on, or outside $\mathbb{T}$, respectively. Then for $T_{\omega^{*}}$ on $H^{p^{\prime}}$, with $1<p^{\prime}<\infty$, we have

$$
\operatorname{Ker}\left(T_{\omega^{*}}\right)=\left\{r_{0} /\left(s_{-}\right)^{\sharp} \mid \operatorname{deg}\left(r_{0}\right)<\operatorname{deg}\left(s_{-}\right)\right\}, \quad \operatorname{Dom}\left(T_{\omega^{*}}\right)=q^{\sharp} H^{p^{\prime}}+\mathcal{P}_{m-1} .
$$

Moreover, we have

$$
\begin{aligned}
& \operatorname{Ran}\left(T_{\omega^{*}}\right)=z^{m-n} s^{\sharp} H^{p^{\prime}}+\widetilde{\mathcal{P}}_{*} \quad \text { if } m \geq n, \\
& \operatorname{Ran}\left(T_{\omega^{*}}\right)=T_{z^{m-n}}\left(s^{\sharp} H^{p^{\prime}}+\widetilde{\mathcal{P}}_{*}\right) \quad \text { if } m<n,
\end{aligned}
$$

where for $m \geq n$ the subspace $\widetilde{\mathcal{P}}_{*}$ is given by

$$
\widetilde{\mathcal{P}}_{*}=\left\{r \in \mathcal{P} \mid r q^{\sharp}=z^{m-n} r_{1} s^{\sharp}+r_{2} \text { for } r_{1}, r_{2} \in \mathcal{P}_{m-1}\right\} \subset \mathcal{P}_{m-n+\operatorname{deg}\left(s^{\sharp}\right)-1} \text {, }
$$


while for $m<n$ we have

$$
\widetilde{\mathcal{P}}_{*}=\left\{r \in \mathcal{P} \mid r q^{\sharp}=r_{1} s^{\sharp}+r_{2} \text { for } r_{1}, r_{2} \in \mathcal{P}_{m-1}\right\} \subset \mathcal{P}_{\operatorname{deg}\left(s^{\sharp}\right)-1} .
$$

Furthermore, $\operatorname{Ran}\left(T_{\omega^{*}}\right)$ is dense in $H^{p^{\prime}}$.

Proof. We separate the cases $m \geq n$ and $m<n$.

For $m \geq n$, we have $\omega^{*}=\widetilde{s} / \widetilde{q} \in \operatorname{Rat}(\mathbb{T})$ with $\widetilde{s}=z^{m-n} s^{\sharp}$ and $\widetilde{q}=q^{\sharp}$. Hence $\widetilde{s}$ factors as $\widetilde{s}=\left(z^{m-n}\left(s_{+}\right)^{\sharp}\right)\left(s_{0}\right)^{\sharp}\left(s_{-}\right)^{\sharp}$, where the factors have all their roots inside, on, or outside $\mathbb{T}$, respectively. Also, $\operatorname{deg}\left(q^{\sharp}\right)=\operatorname{deg}(q)$ and $\operatorname{deg}\left(\left(s_{+}\right)^{\sharp}\right)=\operatorname{deg}\left(s_{+}\right)$. So the formulas for $\operatorname{Dom}\left(T_{\omega^{*}}\right)$ and $\operatorname{Ran}\left(T_{\omega^{*}}\right)$ follow directly from (2.1), while the formula for $\operatorname{Ker}\left(T_{\omega^{*}}\right)$ follows because the bound on the degree of $r_{0}$ can be computed as

$$
m-\operatorname{deg}\left(z^{m-n}\left(s_{+}\right)^{\sharp}\left(s_{0}\right)^{\sharp}\right)=n-\operatorname{deg}\left(\left(s_{+}\right)^{\sharp}\left(s_{0}\right)^{\sharp}\right)=n-\operatorname{deg}\left(s_{+} s_{0}\right)=\operatorname{deg}\left(s_{-}\right) \text {. }
$$

Finally, a complement of the closure of $\operatorname{Ran}\left(T_{\omega^{*}}\right)$ is given by $\mathcal{P}_{k-1}$ with $k$ the maximum of 0 and $\operatorname{deg}\left(z^{m-n}\left(s_{+}\right)^{\sharp}\right)-m=\operatorname{deg}\left(\left(s_{+}\right)^{\sharp}\right)-n \leq 0$. Hence $\mathcal{P}_{-1}=\{0\}$. Thus $T_{\omega^{*}}$ has dense range, as claimed.

In case $m<n$, we have $T_{\omega^{*}}=T_{z^{m-n}} T_{s^{\sharp} / q^{\sharp}}$ and $s^{\sharp} / q^{\sharp}$ is in $\operatorname{Rat}(\mathbb{T})$. Applying the above results for $T_{\omega}$ to $T_{s^{\sharp} / q^{\sharp}}$ directly gives the formulas for $\operatorname{Dom}\left(T_{\omega^{*}}\right)$ and $\operatorname{Ran}\left(T_{\omega^{*}}\right)$.

To see that the formula for $\operatorname{Ker}\left(T_{\omega^{*}}\right)$ holds, we follow the argumentation of the proof of Lemma 4.1 in [4]. For $g \in \operatorname{Dom}\left(T_{\omega^{*}}\right)=\operatorname{Dom}\left(T_{s^{\sharp}} / q^{\sharp}\right)$ to be in $\operatorname{Ker}\left(T_{\omega^{*}}\right)$ is equivalent to $T_{s^{\sharp} / q^{\sharp}} g \in \mathcal{P}_{n-m-1}$. In other words, by Lemma 3.2 in [4], to $s^{\sharp} g=q^{\sharp} \widetilde{r}+r_{1}$ with $r_{1} \in \mathcal{P}_{m-1}$ and $\widetilde{r} \in \mathcal{P}_{n-m-1}$, since then $T_{s^{\sharp} / q^{\sharp}} g=\widetilde{r}$. The latter happens precisely when $g=r /\left(s_{-}\right)^{\sharp}$ with $r \in \mathcal{P}_{\operatorname{deg}\left(s_{-}\right)-1}$. Indeed, in that case $\operatorname{deg}\left(\left(s_{+}\right)^{\sharp}\left(s_{0}\right)^{\sharp} r\right)<n$ which in the equation $\left(s_{+}\right)^{\sharp}\left(s_{0}\right)^{\sharp} r=s^{\sharp} g=q^{\sharp} \widetilde{r}+r_{1}$ corresponds to $\operatorname{deg}(\widetilde{r})<m-1$, as required. Finally, we note that a complement of $\overline{\operatorname{Ran}\left(T_{s^{\sharp} / q^{\sharp}}\right)}$ in $H^{p^{\prime}}$ is given by $\mathcal{P}_{k-1}$ with $k=\max \left\{0, \operatorname{deg} s_{+}^{\sharp}-m\right\} \leq n-m$. Let $f \in H^{p^{\prime}}$ and write $z^{n-m} f=$ $h+r \in \overline{\operatorname{Ran}\left(T_{s^{\sharp} / q^{\sharp}}\right)}+\mathcal{P}_{k-1}$. Then $f=T_{z^{m-n}} z^{n-m} f=T_{z^{m-n}}(h+r)=$ $T_{z^{m-n}} h \in T_{z^{m-n}} \overline{\operatorname{Ran}\left(T_{s^{\sharp} / q^{\sharp}}\right)} \subset \overline{\operatorname{Ran}\left(T_{z^{m-n}} T_{s^{\sharp} / q^{\sharp}}\right)}=\overline{\operatorname{Ran}\left(T_{\omega^{*}}\right)}$. Thus also in this case $\operatorname{Ran}\left(T_{\omega^{*}}\right)$ is dense in $H^{p^{\prime}}$.

We conclude this section with a lemma which will be of use in the sequel.

Lemma 2.2. Let $r_{1}, r_{2} \in \mathcal{P}$. Set $n_{i}=\operatorname{deg}\left(r_{i}\right), i=1,2$, and $n=\operatorname{deg}\left(r_{1}+r_{2}\right)$. Then

$$
\left(r_{1}+r_{2}\right)^{\sharp}=z^{n-n_{1}} r_{1}^{\sharp}+z^{n-n_{2}} r_{2}^{\sharp} .
$$

In case $n<\max \left\{n_{1}, n_{2}\right\}$, then $n_{1}=n_{2}$ and 0 is a root of $r_{1}^{\sharp}+r_{2}^{\sharp}$ with multiplicity $n-n_{1}$, so that the left hand side in the above identity still is a polynomial without a root at 0 .

Proof. By definition, for $z \in \mathbb{T}$ we have

$$
\begin{aligned}
\left(r_{1}+r_{2}\right)^{\sharp}(z) & =z^{n}\left(\overline{r_{1}(1 / \bar{z})}+\overline{r_{2}(1 / \bar{z})}\right)= \\
& =z^{n-n_{1}} z^{n_{1}} \overline{r_{1}(1 / \bar{z})}+z^{n-n_{2}} z^{n_{2}} \overline{r_{2}(1 / \bar{z})} \\
& =z^{n-n_{1}} r_{1}^{\sharp}(z)+z^{n-n_{2}} r_{2}^{\sharp}(z) .
\end{aligned}
$$




\section{The Adjoint of $T_{\omega}$ for $\omega \in \operatorname{Rat}(\mathbb{T})$}

In this section we prove the first main result, Theorem 1.1, for the special case that $\omega \in \operatorname{Rat}(\mathbb{T})$. In this case, the result specializes to the following theorem, which we prove in this section.

Theorem 3.1. Let $\omega=s / q \in \operatorname{Rat}(\mathbb{T})$ with $s, q \in \mathcal{P}$ co-prime and $1<p<\infty$. Set $m=\operatorname{deg}(q), n=\operatorname{deg}(s)$ and let $1<p^{\prime}<\infty$ with $1 / p+1 / p^{\prime}=1$. Then

$$
\operatorname{Dom}\left(T_{\omega}^{*}\right)=q^{\sharp} H^{p^{\prime}} \subset \operatorname{Dom}\left(T_{\omega^{*}}\right) \quad \text { and } \quad T_{\omega}^{*}=\left.T_{\omega^{*}}\right|_{q^{\sharp} H^{p^{\prime}}} \text {. }
$$

In fact, for $g=q^{\sharp} v \in q^{\sharp} H^{p^{\prime}}$ we have $T_{\omega}^{*} g=T_{z^{m-n}} s^{\sharp} v$. Moreover, factorize $s=s_{-} s_{0} s_{+}$with $s_{-}, s_{0}$ and $s_{+}$polynomials having roots only inside, on, or outside $\mathbb{T}$, respectively. Then

$$
\begin{aligned}
& \operatorname{Ran}\left(T_{\omega}^{*}\right)=T_{z^{m-n}} s^{\sharp} H^{p^{\prime}}, \\
& \operatorname{Ker}\left(T_{\omega}^{*}\right)=\left\{\frac{q^{\sharp} r}{\left(s_{-}\right)^{\sharp}} \mid \operatorname{deg}(r)<\operatorname{deg}\left(s_{-}\right)-m\right\} .
\end{aligned}
$$

In particular, we have

$\operatorname{dim} \operatorname{Ker}\left(T_{\omega}^{*}\right)=\max \left\{0, \#\left\{\right.\right.$ zeroes of $\omega^{*}$ outside $\left.\mathbb{T}\right\}-\#\left\{\right.$ poles of $\omega^{*}$ on $\left.\left.\mathbb{T}\right\}\right\}$, where the multiplicities of the zeroes and poles are taken into account. Thus $T_{\omega}^{*}$ is injective if and only if $\omega$ has at least as many poles inside $\mathbb{T}$ as zeroes inside $\mathbb{T}$ unequal to 0 , multiplicities taken into account.

We first present some auxiliary lemmas. Throughout, let $1<p, p^{\prime}<\infty$ such that $1 / p+1 / p^{\prime}=1$. We will consider $T_{\omega}$ as an operator with domain in $H^{p}$ and $T_{\omega^{*}}$ as an operator with domain in $H^{p^{\prime}}$.

Lemma 3.2. Let $\omega=s / q \in \operatorname{Rat}(\mathbb{T})$ with $s, q \in \mathcal{P}$ co-prime, $m=\operatorname{deg}(q)$ and $n=\operatorname{deg}(s)$. Then

$$
q^{\sharp} H^{p^{\prime}} \subset \operatorname{Dom}\left(T_{\omega}^{*}\right) \cap \operatorname{Dom}\left(T_{\omega^{*}}\right) \quad \text { and }\left.\quad T_{\omega}^{*}\right|_{q^{\sharp} H^{p^{\prime}}}=\left.T_{\omega^{*}}\right|_{q^{\sharp} H^{p^{\prime}}} .
$$

Moreover, for $g=q^{\sharp} v \in q^{\sharp} H^{p^{\prime}}$, with $v \in H^{p^{\prime}}$, we have $T_{\omega}^{*} g=T_{z^{m-n}} s^{\sharp} v$, and thus $T_{\omega}^{*}\left(q^{\sharp} H^{p^{\prime}}\right)=T_{z^{m-n}} s^{\sharp} H^{p^{\prime}}$.

Proof. The inclusion $q^{\sharp} H^{p^{\prime}} \subset \operatorname{Dom}\left(T_{\omega^{*}}\right)$ follows from Proposition 2.1. Let $g$ be in $q^{\sharp} H^{p^{\prime}}$, say $g(z)=q^{\sharp}(z) v(z)$ for $v \in H^{p^{\prime}}$. We show that for $f \in \operatorname{Dom}\left(T_{\omega}\right)$ we have $\left\langle T_{w} f, g\right\rangle_{p, p^{\prime}}=\left\langle f, T_{\omega^{*}} g\right\rangle_{p, p^{\prime}}$. Let $f \in \operatorname{Dom}\left(T_{\omega}\right)$ and $h=T_{\omega} f \in H^{p}$, i.e., $s f=q h+r$ for some $r \in \mathcal{P}_{m-1}$, by [4, Lemma 2.3]. Then

$$
\begin{aligned}
\left\langle T_{\omega} f, g\right\rangle_{p, p^{\prime}} & =\left\langle h, q^{\sharp} v\right\rangle_{p, p^{\prime}}=\left\langle h, z^{m} \bar{q} v\right\rangle_{p, p^{\prime}}=\left\langle q h, z^{m} v\right\rangle_{p, p^{\prime}}=\left\langle s f-r, z^{m} v\right\rangle_{p, p^{\prime}} \\
& \left.=\left\langle s f, z^{m} v\right\rangle_{p, p^{\prime}} \quad \text { (because } \operatorname{deg}(r)<m, v \in H^{p^{\prime}}\right) \\
& =\left\langle f, z^{m} \bar{s} v\right\rangle_{p, p^{\prime}}=\left\langle f, z^{m-n} s^{\sharp} v\right\rangle_{p, p^{\prime}} \\
& \left.=\left\langle f, T_{z^{m-n}} s^{\sharp} v\right\rangle_{p, p^{\prime}} \quad \text { (because } f \in H^{p}\right) .
\end{aligned}
$$

It remains to show that $T_{\omega^{*}} g=T_{z^{m-n}} s^{\sharp} v$. If $m \geq n$, then $\omega^{*}=z^{m-n} s^{\sharp} / q^{\sharp}$ is in $\operatorname{Rat}(\mathbb{T})$ and $\omega^{*} g=z^{m-n} s^{\sharp} v \in H^{p^{\prime}}$, so that, $T_{\omega^{*}} g=z^{m-n} s^{\sharp} v=T_{z^{m-n}} s^{\sharp} v$, by Lemma 2.3 in [4]. If $m<n$, we have $T_{\omega^{*}} g=T_{z^{m-n}} T_{s^{\sharp} / q^{\sharp}} g=T_{z^{m-n}}$ $s^{\sharp} v$. 
Lemma 3.3. Let $\omega=s / q \in \operatorname{Rat}(\mathbb{T})$ with $s, q \in \mathcal{P}$ co-prime, $m=\operatorname{deg}(q)$ and $n=\operatorname{deg}(s)$. Let $g \in \operatorname{Dom}\left(T_{\omega}^{*}\right)$ and $k=T_{\omega}^{*} g \in H^{p^{\prime}}$. Then for any $r \in \mathcal{P}_{n-1}$ and $r_{1} \in \mathcal{P}_{m-1}$ so that

$$
s r_{1}=q r+r_{2} \text { for some } r_{2} \in \mathcal{P}_{m-1}
$$

we have

$$
\left\langle r_{1}, k\right\rangle_{p, p^{\prime}}=\langle r, g\rangle_{p, p^{\prime}}
$$

Moreover, we have

$$
z^{m-n} s^{\sharp} g-q^{\sharp} k \in \mathcal{P}_{m-1} \text { if } m \geq n \text { and } s^{\sharp} g-z^{n-m} q^{\sharp} k \in \mathcal{P}_{n-1} \text { if } m<n \text {. }
$$

In particular, $\operatorname{Dom}\left(T_{\omega}^{*}\right) \subset \operatorname{Dom}\left(T_{\omega^{*}}\right)$ and $T_{\omega}^{*}=\left.T_{\omega^{*}}\right|_{\operatorname{Dom}\left(T_{\omega}^{*}\right)}$.

Proof. Let $g \in \operatorname{Dom}\left(T_{\omega}^{*}\right)$ and $k=T_{\omega}^{*} g$. Hence $\left\langle T_{\omega} f, g\right\rangle_{p, p^{\prime}}=\langle f, k\rangle_{p, p^{\prime}}$ for each $f \in \operatorname{Dom}\left(T_{\omega}\right)$. Since $\omega \in \operatorname{Rat}(\mathbb{T})$, we have $\operatorname{Dom}\left(T_{\omega}\right)=q H^{p}+\mathcal{P}_{m-1}$. Let $f=q h+r_{1} \in \operatorname{Dom}\left(T_{\omega}\right)$, with $h \in H^{p}$ and $r_{1} \in \mathcal{P}_{m-1}$. Then $T_{\omega} f=s h+r$ where $r \in \mathcal{P}_{n-1}$ is uniquely determined by (3.3). Thus

$\langle s h, g\rangle+\langle r, g\rangle=\langle s h+r, g\rangle=\left\langle T_{\omega} f, g\right\rangle=\langle f, k\rangle=\left\langle q h+r_{1}, k\right\rangle=\langle q h, k\rangle+\left\langle r_{1}, k\right\rangle$.

We obtain that

$$
\langle s h, g\rangle-\langle q h, k\rangle=\left\langle r_{1}, k\right\rangle-\langle r, g\rangle \text {. }
$$

However, in choosing $f \in \operatorname{Dom}\left(T_{\omega}\right)$ we can choose $h \in H^{p}$ and $r_{1} \in \mathcal{P}_{m-1}$ independently, and in particular set one or the other equal to zero, so that

$$
\begin{aligned}
& \langle s h, g\rangle=\langle q h, k\rangle \quad\left(h \in H^{p}\right), \\
& \left\langle r_{1}, k\right\rangle=\langle r, g\rangle \quad\left(r \in \mathcal{P}_{n-1}, r_{1} \in \mathcal{P}_{m-1} \text { as in }(3.3)\right) .
\end{aligned}
$$

The second identity proves the first claim of the lemma. From the first identity we obtain that

$$
0=\langle h, \bar{s} g-\bar{q} k\rangle_{p, p^{\prime}}=\left\langle h, z^{-n} s^{\sharp} g-z^{-m} q^{\sharp} k\right\rangle_{p, p^{\prime}} \quad\left(h \in H^{p}\right) .
$$

Thus $\mathbb{P}\left(z^{-n} s^{\sharp} g-z^{-m} q^{\sharp} k\right)=0$. On the other hand, for $l=\max \{m, n\}$ we have

$$
z^{l}\left(z^{-n} s^{\sharp} g-z^{-m} q^{\sharp} k\right)=z^{l-n} s^{\sharp} g-z^{l-m} q^{\sharp} k \in H^{p^{\prime}} .
$$

This can only occur if $z^{l-n} s^{\sharp} g-z^{l-m} q^{\sharp} k \in \mathcal{P}_{l-1}$, which proves the second claim.

To complete the proof, we show that $g \in \operatorname{Dom}\left(T_{\omega^{*}}\right)$ and $T_{\omega^{*}} g=k$. For $m \geq n$ we have $\omega^{*} \in \operatorname{Rat}(\mathbb{T})$ and the first inclusion of (3.4) can be rewritten as

$$
\omega^{*} g=\left(\frac{z^{m-n} s^{\sharp}}{q^{\sharp}}\right) g=k+\widetilde{r} / q^{\sharp}, \quad \text { for some } \widetilde{r} \in \mathcal{P}_{m-1} .
$$

Since $\operatorname{deg}\left(q^{\sharp}\right)=\operatorname{deg}(q)=m$, it now follows that $g \in \operatorname{Dom}\left(T_{\omega^{*}}\right)$ and $T_{\omega^{*}} g=k$. In case $m<n$ we have $T_{\omega^{*}}=T_{z^{m-n}} T_{s^{\sharp} / q^{\sharp}}$ and $s^{\sharp} / q^{\sharp} \in \operatorname{Rat}(\mathbb{T})$. Now the second inclusion of (3.4) gives

$$
\left(\frac{s^{\sharp}}{q^{\sharp}}\right) g=z^{n-m} k+\widetilde{r} / q^{\sharp}, \quad \text { for some } \widetilde{r} \in \mathcal{P}_{n-1} \text {. }
$$


Write $\widetilde{r}=\widetilde{r}_{1} q^{\sharp}+\widetilde{r}_{2}$ with $\widetilde{r}_{2} \in \mathcal{P}_{m-1}$. Then $\tilde{r} / q^{\sharp}=\widetilde{r}_{1}+\widetilde{r}_{2} / q^{\sharp}$ and $\operatorname{deg}\left(\widetilde{r}_{1}\right)<$ $m-n$. Since $\widetilde{r}_{2} / q^{\sharp} \in \operatorname{Rat}_{0}(\mathbb{T})$ it follows that $g \in \operatorname{Dom}\left(T_{s^{\sharp}} / q^{\sharp}\right)=\operatorname{Dom}\left(T_{\omega^{*}}\right)$ and $T_{s^{\sharp} / q^{\sharp}} g=z^{n-m} k+\widetilde{r}_{1}$. But then $T_{\omega^{*}} g=T_{z^{m-n}} T_{s^{\sharp}} / q^{\sharp} g=T_{z^{m-n}}\left(z^{n-m} k+\right.$ $\left.\tilde{r}_{1}\right)=k$.

A special case of the following result was proven as part of the proof of Theorem 2.2 in [5].

Lemma 3.4. Let $r, \widetilde{r} \in \mathcal{P}$ be co-prime. Then $r H^{p} \cap \widetilde{r} H^{p}=r \widetilde{r} H^{p}$.

Proof. Let $\widetilde{r} f=r g$ with $f, g \in H^{p}$. Then $f=r \cdot g / \widetilde{r} \in H^{p}$, so we should show $\tilde{f}:=g / \widetilde{r} \in H^{p}$, i.e., $\widetilde{f}$ analytic on $\mathbb{D}$ and $\int_{\mathbb{T}}|\widetilde{f}(z)|^{p} d z<\infty$.

Since $g \in H^{p}$, the function $\tilde{f}$ can only fail to be analytic at the roots of $\widetilde{r}$ inside $\mathbb{D}$. However, if this were the case, then $f=r \widetilde{f}$ would also fail to be analytic in $\mathbb{D}$, since $r$ and $\widetilde{r}$ are co-prime. Thus $\widetilde{f}$ is analytic on $\mathbb{D}$.

Divide $\mathbb{T}$ as $\mathbb{T}_{1} \cup \mathbb{T}_{2}$ with $\mathbb{T}_{1} \cap \mathbb{T}_{2}=\emptyset$ in such a way that $\mathbb{T}_{1}$ and $\mathbb{T}_{2}$ are both nonempty finite unions of line segments of $\mathbb{T}$ so that the interior of $\mathbb{T}_{1}$ contains the roots of $r$ and the interior of $\mathbb{T}_{2}$ the roots of $\widetilde{r}$. Then $|\widetilde{r}(z)|>N_{1}$ on $\mathbb{T}_{1}$ and $|r(z)|>N_{2}$ on $\mathbb{T}_{2}$ for some $N_{1}, N_{2}>0$. Note that $f=r \widetilde{f}$ and $g=\tilde{r} \tilde{f}$. We then obtain

$\int_{\mathbb{T}_{2}}|\tilde{f}(z)|^{p} d z=\int_{\mathbb{T}_{2}}|f(z) / r(z)|^{p} d z \leq N_{2}^{-p} \int_{\mathbb{T}_{2}}|f(z)|^{p} d z \leq\left(2 \pi N_{2}^{p}\right)^{-1}\|f\|_{H^{p}}^{p}$.

Using $g=\tilde{r} \tilde{f}$, one obtains similarly that $\int_{\mathbb{T}_{1}}|\tilde{f}(z)|^{p} d z \leq\left(2 \pi N_{1}^{p}\right)^{-1}\|g\|_{H^{p}}^{p}$. Thus $\int_{\mathbb{T}}|\tilde{f}(z)|^{p} d z<\infty$.

Proof of Theorem 3.1. By Lemma 3.2, in order to prove (3.1), the formula for the action of $T_{\omega}^{*}$ on $q^{\sharp} H^{p^{\prime}}$ and for the range of $T_{\omega}^{*}$ in $(3.2)$, it remains to show that $\operatorname{Dom}\left(T_{\omega}^{*}\right) \subset q^{\sharp} H^{p^{\prime}}$.

View $\mathcal{P}$ and $\mathcal{P}_{k}, k=1,2, \ldots$, as subspaces of $H^{p}$ or $H^{p^{\prime}}$, write $P_{k}$ for the projection onto $\mathcal{P}_{k-1}$ and set $Q_{k}=I-P_{k}$. Also, the standard $k \times k$ compression of a Toeplitz operator $T_{\phi}$ on $H^{p}$ (or $H^{p^{\prime}}$ ) is denoted by $T_{\phi, k}$, i.e., $T_{\phi, k}=\left.P_{k} T_{\phi}\right|_{\mathcal{P}_{k-1}}$. Now, the relation (3.3) between $r \in \mathcal{P}_{n-1}$ and $r_{1} \in \mathcal{P}_{m-1}$ can be rewritten as

$$
T_{s} r_{1}-T_{q} r \in \mathcal{P}_{m-1},
$$

or, equivalently, as

$$
Q_{m} T_{s} P_{m} r_{1}=Q_{m} T_{s} r_{1}=Q_{m} T_{q} r=Q_{m} T_{q} P_{n} r .
$$

We now consider the cases $m \geq n$ and $m<n$ separately.

First assume $m \geq n$. We can then decompose $Q_{m} T_{s} P_{m}$ and $Q_{m} T_{q} P_{n}$ as

$$
\begin{aligned}
Q_{m} T_{s} P_{m} & =\left[\begin{array}{cc}
0 T_{s^{\sharp}, n}^{*} T_{z^{m-n}}^{*} \\
0 & 0
\end{array}\right]: \mathcal{P}_{m-1}=\left[\begin{array}{c}
\mathcal{P}_{m-n} \\
T_{z^{m-n}} \mathcal{P}_{n-1}
\end{array}\right] \rightarrow\left[\begin{array}{c}
\mathcal{P}_{n-1} \\
T_{z}^{n} H^{p}
\end{array}\right], \\
Q_{m} T_{q} P_{n} & =\left[\begin{array}{c}
T_{q^{\sharp}, n}^{*} \\
0
\end{array}\right]: \mathcal{P}_{n-1} \rightarrow\left[\begin{array}{c}
\mathcal{P}_{n-1} \\
T_{z^{n}} H^{p}
\end{array}\right] .
\end{aligned}
$$

Hence, in this case the identity in (3.5) can be written as

$$
T_{s^{\sharp}, n}^{*}\left(T_{z^{m-n}}^{*} r_{1}\right)=T_{q^{\sharp}, n}^{*} r .
$$


Since all Toeplitz matrices are upper triangular, we in fact have

$$
T_{s^{\sharp}, m}^{*} T_{z^{m-n}, m}^{*} r_{1}=T_{q^{\sharp}, m}^{*} r .
$$

Note that $T_{q^{\sharp}, n}^{*}$ is invertible, because $q$ has only roots on $\mathbb{T}$ so that $q(0) \neq 0$. We obtain that for given $r_{1} \in \mathcal{P}_{m-1}$, the polynomial $r \in \mathcal{P}_{n-1}$ that satisfies (3.3) is uniquely determined by

$$
r=\left(T_{q^{\sharp}, m}^{*}\right)^{-1} T_{s^{\sharp}, m}^{*} T_{z^{m-n}, m}^{*} r_{1}=T_{s^{\sharp}, m}^{*} T_{z, m}^{* m-n}\left(T_{q^{\sharp}, m}^{*}\right)^{-1} r_{1},
$$

where the commutation of Toeplitz matrices can occur since they all have analytic symbols. Now take $r_{1} \in \mathcal{P}_{m-1}$ arbitrary, and define $r$ as above, so that (3.3) holds. Then, by Lemma 3.3, we have

$$
\begin{aligned}
\left\langle r_{1}, P_{m} k\right\rangle_{\mathcal{P}_{m-1}} & =\left\langle r_{1}, k\right\rangle_{p, p^{\prime}}=\langle r, g\rangle_{p, p^{\prime}}=\left\langle r, P_{m} g\right\rangle_{\mathcal{P}_{m-1}} \\
& =\left\langle T_{s^{\sharp}, m}^{*} T_{z, m}^{* m-n}\left(T_{q^{\sharp}, m}^{*}\right)^{-1} r_{1}, P_{m} g\right\rangle_{\mathcal{P}_{m-1}} \\
& =\left\langle r_{1},\left(T_{q^{\sharp}, m}\right)^{-1} T_{z, m}^{m-n} T_{s^{\sharp}, m} P_{m} g\right\rangle_{\mathcal{P}_{m-1}} .
\end{aligned}
$$

Since $r_{1} \in \mathcal{P}_{m-1}$ is arbitrary, we have $P_{m} k=\left(T_{q^{\sharp}, m}\right)^{-1} T_{z, m}^{m-n} T_{s^{\sharp}, m} P_{m} g$, and thus

$$
P_{m} T_{q^{\sharp}} k=T_{q^{\sharp}, m} P_{m} k=T_{z, m}^{m-n} T_{s^{\sharp}, m} P_{m} g=P_{m} T_{z}^{m-n} T_{s^{\sharp}} g .
$$

This shows that $P_{m} q^{\sharp} k=P_{m} z^{m-n} s^{\sharp} g$. Together with the first inclusion in (3.4) we obtain that

$$
q^{\sharp} k=z^{m-n} s^{\sharp} g \text {. }
$$

Since $q^{\sharp}$ and $z^{m-n} s^{\sharp}$ are co-prime, we can apply Lemma 3.4 to conclude $g \in q^{\sharp} H^{p^{\prime}}$.

Now assume $m<n$. By [4, Lemma 2.4], we can write $\omega=\omega_{0}+\omega_{1}$ uniquely with $\omega_{0} \in \operatorname{Rat}_{0}(\mathbb{T})$ and $\omega_{1} \in$ Rat without poles on $\mathbb{T}$, i.e, $\omega_{1} \in$ $L^{\infty}(\mathbb{T})$. In fact $\omega_{1} \in \mathcal{P}$, since all poles of $\omega$ are on $\mathbb{T}$, and $\omega_{0}=\widetilde{s} / q$ with $\tilde{s} \in \mathcal{P}_{m-1}$. It now follows that $\operatorname{Dom}\left(T_{\omega_{0}}^{*}\right)=q^{\sharp} H^{p^{\prime}}$, and since $T_{\omega_{1}}$ is bounded, $\operatorname{Dom}\left(T_{\omega}^{*}\right)=\operatorname{Dom}\left(T_{\omega_{0}}^{*}\right)=q^{\sharp} H^{p^{\prime}}$. Furthermore, $T_{\omega}^{*}=T_{\omega_{0}}^{*}+\left.T_{\omega_{1}}^{*}\right|_{q^{\sharp} H^{p^{\prime}}}=$ $\left.T_{\omega_{0}^{*}}\right|_{q^{\sharp} H^{p^{\prime}}}+\left.T_{\omega_{1}^{*}}\right|_{q^{\sharp} H^{p^{\prime}}}=\left.T_{\omega^{*}}\right|_{q^{\sharp} H^{p^{\prime}}}$.

In the next part of the proof we prove the formula for $\operatorname{Ker}\left(T_{\omega^{*}}\right)$, without distinguishing between the proper and non-proper case. Let $g=q^{\sharp} v \in$ $\operatorname{Dom}\left(T_{\omega}^{*}\right)$ with $v \in H^{p^{\prime}}$. Then $g \in \operatorname{Ker}\left(T_{\omega}^{*}\right)$ if and only if $g \in \operatorname{Ker}\left(T_{\omega^{*}}\right)$, i.e., $g=q^{\sharp} v=r_{1} /\left(s_{-}\right)^{\sharp}$ for $r_{1} \in \mathcal{P}_{\operatorname{deg}\left(s_{-}\right)-1}$, see Proposition 2.1. Thus $v=r_{1} /\left(\left(s_{-}\right)^{\sharp} q^{\sharp}\right) \in \operatorname{Rat} \cap H^{p^{\prime}}$. Then $v \in H^{p^{\prime}}$ implies $r_{1}=q^{\sharp} r$, and $\operatorname{deg}(r)=$ $\operatorname{deg}\left(r_{1}\right)-m<\operatorname{deg}\left(s_{-}\right)-m$. Hence $g=q^{\sharp} r /\left(s_{-}\right)^{\sharp}$ with $\operatorname{deg}(r)<\operatorname{deg}\left(s_{-}\right)-m$. That all such functions are in $\operatorname{Ker}\left(T_{\omega}^{*}\right)=\operatorname{Ker}\left(T_{\omega^{*}}\right) \cap q^{\sharp} H^{p^{\prime}}$ follows directly from the formula for $\operatorname{Ker}\left(T_{\omega^{*}}\right)$ obtained in Proposition 2.1. The formula for the dimension of $\operatorname{Ker}\left(T_{\omega}^{*}\right)$ follows directly and the condition for injectivity follows since $\operatorname{deg}\left(s_{-}\right)^{\sharp}$ is equal to the number of nonzero roots of $s_{-}$, counting multiplicity. 


\section{The Adjoint of $T_{\omega}$ : General Case}

In the section we prove Theorem 1.1 in full generality. Hence let $\omega=s / q \in$ Rat with $s, q \in \mathcal{P}$ co-prime. As in Theorem 1.1, factor $s=s_{-} s_{0} s_{+}$and $q=q_{-} q_{0} q_{+}$with $s_{-}, q_{-}$having roots only inside $\mathbb{T}, s_{0}, q_{0}$ having roots only on $\mathbb{T}$, and $s_{+}, q_{+}$having roots only outside $\mathbb{T}$. Set $m=\operatorname{deg}(q), n=\operatorname{deg}(s)$, $m_{ \pm}=\operatorname{deg}\left(q_{ \pm}\right), n_{ \pm}=\operatorname{deg}\left(s_{ \pm}\right)$, and $m_{0}=\operatorname{deg}\left(q_{0}\right), n_{0}=\operatorname{deg}\left(s_{0}\right)$. By Lemma 5.1 in [4], and its proof, we can factor $\omega$ as $\omega=\omega_{-}\left(z^{\kappa} \omega_{0}\right) \omega_{+}$with $\kappa=$ $n_{-}-m_{-}, \omega_{-}=s_{-} /\left(z^{\kappa} q_{-}\right)$having only poles and zeroes inside $\mathbb{T}, \omega_{0}=s_{0} / q_{0}$ having only poles and zeroes on $\mathbb{T}$, and $\omega_{+}=s_{+} / q_{+}$having only poles and zeroes outside $\mathbb{T}$, and we have $T_{\omega}=T_{\omega_{-}} T_{z^{\kappa} \omega_{0}} T_{\omega_{+}}$. Moreover, $T_{\omega_{-}}$and $T_{\omega_{+}}$ are bounded and boundedly invertible.

Note that $T_{\omega_{-}} T_{z^{\kappa} \omega_{0}}$ is closed and densely defined and $\operatorname{Ran}\left(T_{\omega_{+}}\right)=H^{p}$, and thus by Corollary 1 in [12]

$$
T_{\omega}^{*}=T_{\omega_{+}}^{*}\left(T_{\omega_{-}} T_{z^{\kappa} \omega_{0}}\right)^{*} .
$$

Furthermore, $T_{\omega_{-}}$is bounded and $T_{z^{\kappa} \omega_{0}}$ is closed and densely defined. By Theorem 4 in [13] one has

$$
\left(T_{\omega_{-}} T_{z^{\kappa} \omega_{0}}\right)^{*}=T_{z^{\kappa} \omega_{0}}^{*} T_{\omega_{-}}^{*} .
$$

Combining this and using that $T_{\omega_{+}}^{*}=T_{\omega_{+}^{*}}$ and $T_{\omega_{-}}^{*}=T_{\omega_{-}^{*}}$ we see that

$$
T_{\omega}^{*}=T_{\omega_{+}}^{*} T_{z^{\kappa} \omega_{0}}^{*} T_{\omega_{-}}^{*}=T_{\omega_{+}^{*}} T_{z^{\kappa} \omega_{0}}^{*} T_{\omega_{-}^{*}} \quad \text { on } \operatorname{Dom}\left(T_{\omega}^{*}\right) .
$$

Note that

$$
\begin{aligned}
\omega_{-}^{*} & =\frac{\left(s_{-}\right)^{\sharp}}{\left(q_{-}\right)^{\sharp}}, \quad \omega_{0}^{*}=z^{m_{0}-n_{0}} \frac{\left(s_{0}\right)^{\sharp}}{\left(q_{0}\right)^{\sharp}}, \\
\left(z^{\kappa} \omega_{0}\right)^{*} & =z^{m_{0}-n_{0}-\kappa} \frac{\left(s_{0}\right)^{\sharp}}{\left(q_{0}\right)^{\sharp}}, \quad \omega_{+}^{*}=z^{m_{+}-n_{+}} \frac{\left(s_{+}\right)^{\sharp}}{\left(q_{+}\right)^{\sharp}} .
\end{aligned}
$$

By construction, $\omega_{-}$and $1 / \omega_{-}$are both anti-analytic. Consequently, $\omega_{-}^{*}$ and $1 / \omega_{-}^{*}$ are both analytic functions. This implies $T_{\omega_{-}^{*}}^{ \pm}\left(q_{0}\right)^{\sharp} H^{p^{\prime}} \subset\left(q_{0}\right)^{\sharp} H^{p^{\prime}}$, and thus $T_{\omega_{-}^{*}}\left(q_{0}\right)^{\sharp} H^{p^{\prime}}=\left(q_{0}\right)^{\sharp} H^{p^{\prime}}$. Since $T_{\omega_{+}^{*}}$ is invertible, to see that $\operatorname{Dom}\left(T_{\omega}^{*}\right)=$ $\left(q_{0}\right)^{\sharp} H^{p^{\prime}}$ it suffices to show $\operatorname{Dom}\left(T_{z^{\kappa} \omega_{0}}^{*}\right)=\left(q_{0}\right)^{\sharp} H^{p^{\prime}}$. For the case where $\kappa \geq 0$, so that $z^{\kappa} \omega_{0} \in \operatorname{Rat}(\mathbb{T})$, this follows directly from Theorem 3.1. For $\kappa<0$, note that $T_{z^{\kappa} \omega_{0}}=T_{z^{\kappa}} T_{\omega_{0}}$, so that $T_{z^{\kappa} \omega_{0}}^{*}=T_{\omega_{0}}^{*} T_{z^{\kappa}}^{*}=T_{\omega_{0}}^{*} T_{z^{-\kappa}}$, again using Theorem 4 of [13]. Then $g \in \operatorname{Dom}\left(T_{z^{\kappa} \omega_{0}}^{*}\right)$ holds if and only if $z^{-\kappa} g \in \operatorname{Dom}\left(T_{\omega_{0}}^{*}\right)=\left(q_{0}\right)^{\sharp} H^{p^{\prime}}$. By Lemma 3.4 this is the same as $g \in$ $\left(q_{0}\right)^{\sharp} H^{p^{\prime}}$, since $z^{-\kappa}$ and $q_{0}^{\sharp}$ are co-prime. Thus in both cases we arrive at $\operatorname{Dom}\left(T_{\omega}^{*}\right)=\left(q_{0}\right)^{\sharp} H^{p^{\prime}}$. Moreover, we also find that $T_{z^{\kappa} \omega_{0}}^{*}=\left.T_{\left(z^{\kappa} \omega_{0}\right)^{*}}\right|_{\left(q_{0}\right)^{\sharp} H^{p^{\prime}}}$, so that

$$
T_{\omega}^{*}=T_{\omega_{+}^{*}} T_{z^{\kappa} \omega_{0}}^{*} T_{\omega_{-}^{*}}=\left.T_{\omega_{+}^{*}} T_{\left(z^{\kappa} \omega_{0}\right)^{*}} T_{\omega_{-}^{*}}\right|_{\left(q_{0}\right)^{\sharp} H^{p^{\prime}}}=\left.T_{\omega^{*}}\right|_{\left(q_{0}\right)^{\sharp} H^{p^{\prime}}} .
$$

Hence (1.2) holds.

Next we derive the formula for $\operatorname{Ker}\left(T_{\omega}^{*}\right)$. For $\kappa \geq 0$ we have $g \in \operatorname{Ker}\left(T_{\omega}^{*}\right)$ if and only if $T_{\omega_{-}^{*}} g \in \operatorname{Ker}\left(T_{z^{\kappa} \omega_{0}}^{*}\right)=\left(q_{0}\right)^{\sharp} \mathcal{P}_{\kappa-m_{0}-1}$, where the last identity follows by applying Theorem 3.1 to $z^{\kappa} \omega_{0}$. Thus $g \in \operatorname{Ker}\left(T_{\omega}^{*}\right)$ if and only if $\left(\left(s_{-}\right)^{\sharp} /\left(q_{-}\right)^{\sharp}\right) g=\left(q_{0}\right)^{\sharp} r$, i.e., $g=\left(q_{-}\right)^{\sharp}\left(q_{0}\right)^{\sharp} r /\left(s_{-}\right)^{\sharp}$, for some $r \in \mathcal{P}_{\kappa-m_{0}-1}$, 
as claimed. For $\kappa<0$ we have $g \in \operatorname{Ker}\left(T_{\omega}^{*}\right)$ if and only if $z^{-\kappa} \omega_{-}^{*} g \in \operatorname{Ker}\left(T_{\omega_{0}}^{*}\right)$. However, $\operatorname{Ker}\left(T_{\omega_{0}}^{*}\right)=\{0\}$, by Theorem 3.1, so that $\operatorname{Ker}\left(T_{\omega}^{*}\right)=\{0\}$, in line with the formula in (1.3). The formula for the dimension of $\operatorname{Ker}\left(T_{\omega}^{*}\right)$ follows directly.

Now we turn to the formula for $\operatorname{Ran}\left(T_{\omega}^{*}\right)$. Note that

$$
\operatorname{Ran}\left(T_{\omega}^{*}\right)=T_{\omega_{+}^{*}} \operatorname{Ran}\left(T_{z^{\kappa} \omega_{0}}^{*} T_{\omega_{-}^{*}}\right)=T_{\omega_{+}^{*}} \operatorname{Ran}\left(T_{z^{\kappa} \omega_{0}}^{*}\right) .
$$

We first show that $\operatorname{Ran}\left(T_{z^{\kappa} \omega_{0}}^{*}\right)=T_{z^{m_{0}-n_{0}-\kappa}}\left(s_{0}\right)^{\sharp} H^{p^{\prime}}$. Again, for the case $\kappa \geq 0$ this follows directly from Theorem 3.1. Assume $\kappa<0$. Then $T_{z^{\kappa} \omega_{0}}^{*}=$ $T_{\omega_{0}}^{*} T_{z^{-\kappa}}$. Hence,

$$
\begin{aligned}
\operatorname{Ran}\left(T_{z^{\kappa} \omega_{0}}^{*}\right) & =T_{\omega_{0}}^{*}\left(z^{-\kappa} H^{p^{\prime}} \cap \operatorname{Dom}\left(T_{\omega_{0}}\right)\right)=T_{\omega_{0}}^{*}\left(z^{-\kappa} H^{p^{\prime}} \cap\left(q_{0}\right)^{\sharp} H^{p^{\prime}}\right) \\
& =T_{\omega_{0}}^{*} z^{-\kappa}\left(q_{0}\right)^{\sharp} H^{p^{\prime}} .
\end{aligned}
$$

The last identity follows by Lemma 3.4. Now the action of $T_{\omega_{0}}^{*}$, as described in Theorem 3.1, shows that $\operatorname{Ran}\left(T_{z^{\kappa} \omega_{0}}^{*}\right)=T_{z^{m_{0}-n_{0}}} z^{-\kappa}\left(s_{0}\right)^{\sharp} H^{p^{\prime}}=$ $T_{z^{m_{0}-n_{0}-\kappa}}\left(s_{0}\right)^{\sharp} H^{p^{\prime}}$. Since $1 / q_{+}$is analytic, $1 /\left(q_{+}\right)^{\sharp}$ is anti-analytic, and therefore, independent of the sign of $m_{+}-n_{+}$, we have

$$
T_{\omega_{+}^{*}}=T_{1 /\left(q_{+}\right)^{\sharp}} T_{z^{m_{+}-n_{+}}} T_{\left(s_{+}\right)^{\sharp}} \text {. }
$$

Thus

$$
\operatorname{Ran}\left(T_{\omega}^{*}\right)=T_{1 /\left(q_{+}\right)^{\sharp}} T_{z^{m_{+}-n_{+}}} T_{\left(s_{+}\right)^{\sharp}} T_{z^{m_{0}-n_{0}-\kappa}}\left(s_{0}\right)^{\sharp} H^{p^{\prime}} .
$$

Note that $T_{\left(s_{+}\right)^{\sharp}}$ and $T_{z^{m_{0}-n_{0}-\kappa}}$ need not commute, in case $m_{0}-n_{0}-\kappa<0$. However, we do have $T_{\left(s_{+}\right)^{\sharp}} T_{z^{m_{0}-n_{0}-\kappa}}=T_{z^{m_{0}-n_{0}-\kappa}} T_{\left(s_{+}\right)^{\sharp}} Q_{\kappa+n_{0}-m_{0}}$. Moreover, since $\left(s_{+}\right)^{\sharp}$ is analytic, $T_{\left(s_{+}\right)^{\sharp}} Q_{\kappa+n_{0}-m_{0}}=Q_{\kappa+n_{0}-m_{0}} T_{\left(s_{+}\right)^{\sharp}} Q_{\kappa+n_{0}-m_{0}}$ and we have

$$
\begin{aligned}
T_{z^{m_{+}-n_{+}}} T_{z^{m_{0}-n_{0}-\kappa}} Q_{\kappa+n_{0}-m_{0}} & =T_{z^{m_{+}-n_{+}+m_{0}-n_{0}-\kappa}} Q_{\kappa+n_{0}-m_{0}} \\
& =T_{z^{m-n}} Q_{\kappa+n_{0}-m_{0}} .
\end{aligned}
$$

Therefore, we have

$$
\begin{aligned}
\operatorname{Ran}\left(T_{\omega}^{*}\right) & =T_{1 /\left(q_{+}\right)^{\sharp}} T_{z^{m-n}} T_{\left(s_{+}\right)^{\sharp}} Q_{\kappa+n_{0}-m_{0}}\left(s_{0}\right)^{\sharp} H^{p^{\prime}} \\
& =T_{z^{m-n}\left(s_{+}\right)^{\sharp} /\left(q_{+}\right)^{\sharp}} Q_{\kappa+n_{0}-m_{0}}\left(s_{0}\right)^{\sharp} H^{p^{\prime}},
\end{aligned}
$$

again using that $1 /\left(q_{+}\right)^{\sharp}$ is anti-analytic and $\left(s_{+}\right)^{\sharp}$ is analytic. This gives the general formula for $\operatorname{Ran}\left(T_{\omega}^{*}\right)$. In case $\kappa+n_{0}-m_{0} \leq 0$, we have $Q_{\kappa+n_{0}-m_{0}}=I$ and $T_{\left(s_{+}\right)^{\sharp}} Q_{\kappa+n_{0}-m_{0}}\left(s_{0}\right)^{\sharp}=\left(s_{+} s_{0}\right)^{\sharp}$, as claimed.

\section{Symmetric Operators and Selfadjoint Extensions}

For $\omega \in$ Rat, the second adjoint $T_{\omega}^{* *}$ is well-defined and $T_{\omega}^{* *}=T_{\omega}$, since $T_{\omega}$ is a closed, densely defined operator on a reflexive Banach space $[7$, Theorem III.5.24]. Now consider $\omega \in \operatorname{Rat}(\mathbb{T})$ and $p=2$. From Theorem 1.1 it is obvious that $T_{\omega} \neq T_{\omega}^{*}$, except in the degenerate case where $q$ is constant, since $\operatorname{Dom}\left(T_{\omega}\right)=q H^{2}+\mathcal{P}_{\operatorname{deg}(q)-1}$ contains all polynomials while $\operatorname{Dom}\left(T_{\omega}^{*}\right)=q^{\sharp} H^{2}$ only contains the polynomials that contain $q^{\sharp}$ as a factor. Consequently, $T_{\omega}$ cannot be selfadjoint. In this section we consider the question when $T_{\omega}^{*}$ is 
symmetric, and, if this is the case, when does $T_{\omega}^{*}$ have a selfadjoint extension $L$. The first topic is addressed in the following theorem.

Theorem 5.1. Let $\omega=s / q \in \operatorname{Rat}(\mathbb{T})$ with $s, q \in \mathcal{P}$ co-prime. Set $n=\operatorname{deg}(s)$ and $m=\operatorname{deg}(q)$. Then the following are equivalent.

(1) $T_{\omega}^{*}$ is symmetric;

(2) $\omega(\mathbb{T}) \subset \mathbb{R}$;

(3) $\omega(z)=\widetilde{\omega}\left(-i \frac{z+1}{z-1}\right)$ with $\widetilde{\omega}$ a real rational function with poles only on $\mathbb{R}$;

(4) the essential spectrum $\sigma_{\text {ess }}\left(T_{\omega}\right)$ of $T_{\omega}$ is contained in $\mathbb{R}$;

(5) $\omega$ is proper, $s=z^{m-n} \widetilde{s}$ with $\widetilde{s}$ self-inversive and $q_{0} \overline{s_{n}}=\overline{q_{m}} s_{m-n}$ holds, where $s(z)=\sum_{k=0}^{n} s_{k} z^{k}$ and $q(z)=\sum_{k=0}^{m} q_{k} z^{k}$.

Moreover, if $T_{\omega}^{*}$ is symmetric, then $T_{\omega}^{*} \subset T_{\omega}$.

Proof. We first prove the equivalence of (1) and (2), and that (1) implies $T_{\omega}^{*} \subset$ $T_{\omega}$. Assume (2). For $z \in \mathbb{T}$, not a root of $q$, we have $\omega^{*}(z)=\overline{\omega(z)}=\omega(z)$. Hence $\omega^{*}=\omega$. Since $q$ has only roots on $\mathbb{T}$, we have $q=\gamma q^{\sharp}$ for a unimodular constant $\gamma$. Hence $q H^{2}=q^{\sharp} H^{2}$. This shows $T_{\omega}^{*}=\left.T_{\omega^{*}}\right|_{q^{\sharp} H^{2}}=\left.T_{\omega}\right|_{q H^{2}} \subset T_{\omega}$. Since $\left(T_{\omega}^{*}\right)^{*}=T_{\omega}$, it follows that $T_{\omega}^{*}$ is symmetric and $T_{\omega}^{*} \subset T_{\omega}$. Conversely, assume (1). Then we still have $q H^{2}=q^{\sharp} H^{2}$ and $T_{\omega}^{*} \subset\left(T_{\omega}^{*}\right)^{*}=T_{\omega}$. Hence $T_{\omega}^{*}=\left.T_{\omega}\right|_{q H^{2}}$. In particular, we have $\omega^{*} q=T_{\omega^{*}} q=T_{\omega}^{*} q=T_{\omega} q=\omega q$. This implies $\omega=\omega^{*}$. Hence $\omega(z)=\overline{\omega(z)}$ for $z \in \mathbb{T}$, not a root of $q$. Thus $\omega(\mathbb{T}) \subset \mathbb{R}$.

That (2) and (3) are equivalent follows simply because in (3) $\omega$ is the composition of $\widetilde{\omega}$ and the inverse Cayley transform, which maps the circle $\mathbb{T}$ bijectively onto $\mathbb{R}$. The fact that $\widetilde{\omega}$ is real rational, i.e., $\widetilde{\omega}=\widetilde{s} / \widetilde{q}$ with $\widetilde{s}$ and $\widetilde{q}$ real polynomials, is equivalent to $\widetilde{\omega}(\mathbb{R}):=\{\widetilde{\omega}(t): t \in \mathbb{R}, \widetilde{q}(t) \neq 0\} \subset \mathbb{R}$. Also, the equivalence of (2) and (4) is a direct consequence of the fact that $\sigma_{\text {ess }}\left(T_{\omega}\right)=\omega(\mathbb{T})$, by $[5$, Theorem 1.1$]$.

Finally, we prove $(2) \Leftrightarrow(5)$. Since $q=\gamma q^{\sharp}$, we have

$$
\omega^{*}=z^{m-n} \frac{s^{\sharp}}{q^{\sharp}}=z^{m-n} \gamma \frac{s^{\sharp}}{q} .
$$

Thus, we have $\omega=\omega^{*}$ if and only if $z^{m-n} \gamma s^{\sharp}=s$. Hence (2) is equivalent to $z^{m-n} \gamma s^{\sharp}=s$. Now assume (2). Since $\operatorname{deg}\left(s^{\sharp}\right) \leq \operatorname{deg}(s)$, the identity $z^{m-n} \gamma s^{\sharp}=s$ can only occur if $m \geq n$, i.e., if $\omega$ is proper. The identity also shows that $s=z^{m-n} \widetilde{s}$ for $\widetilde{s}=\gamma s^{\sharp}$. On the other hand, $s^{\sharp}=$ $\left(z^{m-n} \widetilde{s}\right)^{\sharp}=\widetilde{s}^{\sharp}$. Thus $\widetilde{s}=\gamma s^{\sharp}=\gamma \widetilde{s}^{\sharp}$, which shows $\widetilde{s}$ is self-inversive, with constant $\gamma$. Note that $\gamma=q_{0} / \overline{q_{m}}$. Also, we have $s_{0}=\cdots=s_{m-n-1}=0$ and $\widetilde{s}(z)=\sum_{k=0}^{2 n-m} s_{m-n+k} z^{k}$. Since $\widetilde{s}$ is self-inversive, $\widetilde{s}=\delta \widetilde{s}^{\sharp}$ with $\delta=s_{m-n} / \overline{s_{n}}$. But also $\delta=\gamma$, so $s_{m-n} / \overline{s_{n}}=q_{0} / \overline{q_{m}}$. Thus $q_{0} \overline{s_{n}}=\overline{q_{m}} s_{m-n}$. Hence $(5)$ holds. Conversely, assume (5). Reversing the above argument, it follows that $q_{0} \overline{s_{n}}=\overline{q_{m}} s_{m-n}$ implies $\widetilde{s}=\delta \widetilde{s}^{\sharp}$ with $\delta=\gamma$. Thus $\gamma s^{\sharp}=\gamma \widetilde{s}^{\sharp}=\widetilde{s}$. This implies $s=z^{m-n} \widetilde{s}=z^{m-n} \gamma s^{\sharp}$, and hence (2).

Corollary 5.2. Let $\omega=s / q \in \operatorname{Rat}(\mathbb{T})$ with $s, q \in \mathcal{P}$ co-prime.Assume $T_{\omega}^{*}$ is symmetric. Then $\operatorname{deg}(s) \leq \operatorname{deg}(q) \leq 2 \operatorname{deg}(s)$. 
Proof. By Theorem 5.1 condition (5) holds with $m=\operatorname{deg}(q)$ and $n=\operatorname{deg}(s)$. Since $\widetilde{s}$ is self-inversive, we have $\widetilde{s}(0) \neq 0$. Consequently, 0 would be a nonremovable singularity of $s=z^{m-n} \widetilde{s}$ in case $m<n$, which gives a contradiction. Hence $m \geq n$. Furthermore, comparing the degrees on both sides of $s=z^{m-n} \widetilde{s}$ yields, $n=m-n+\operatorname{deg}(\widetilde{s}) \geq m-n$. Hence $m \leq 2 n$.

When $T_{\omega}^{*}$ is symmetric, it need not be the case that $T_{\omega}^{*}$ has a selfadjoint extension. In Proposition 5.4 below we characterize when $T_{\omega}^{*}$ does have a selfadjoint extension. However, we first give a concrete example that shows this does not always happen.

Example 5.3. In [6] Helson considered the functions $\omega_{k}(z)=\left(-i \frac{z+1}{z-1}\right)^{k}$ for $k \in \mathbb{N}$. For all $k$ we have $\omega_{k}(\mathbb{T}) \subset \mathbb{R}$, see Theorem $5.1(3)$ above, hence $T_{\omega_{k}}^{*}$ is symmetric by Theorem 5.1. In fact, for $k$ even $\omega_{k}(\mathbb{T})=\mathbb{R}_{+}$, while for $k$ odd we have $\omega_{k}(\mathbb{T})=\mathbb{R}$. We show that $T_{\omega_{k}}^{*}$ does not have a selfadjoint extension for $k=1$. In Example 5.8 we return to this example for general $k$.

For $k=1$ we have $\omega(z)=\omega_{1}(z)=-i \frac{z+1}{z-1}$. Hence $\operatorname{Dom}\left(T_{\omega}\right)=(z-$ 1) $H^{2}+\mathbb{C}$ and $\operatorname{Dom}\left(T_{\omega}^{*}\right)=(z-1) H^{2}$. Suppose $T_{\omega}^{*}$ has a selfadjoint extension $L$. Then $L=L^{*}$ and thus $T_{\omega}^{*} \subset L=L^{*} \subset T_{\omega}^{* *}=T_{\omega}$. Since $T_{\omega}$ is not selfadjoint, the inclusions are strict. Hence $\operatorname{Dom}\left(T_{\omega}^{*}\right) \subset \operatorname{Dom}(L) \subset \operatorname{Dom}\left(T_{\omega}\right)$, with strict inclusions. However, the complement of $\operatorname{Dom}\left(T_{\omega}^{*}\right)$ in $\operatorname{Dom}\left(T_{\omega}\right)$ is one-dimensional, hence not both inclusions can be strict. Thus $T_{\omega}$ does not admit a selfadjoint extension.

Proposition 5.4. Let $\omega=s / q \in \operatorname{Rat}(\mathbb{T})$, with $s, q \in \mathcal{P}$ coprime, be such that $T_{\omega}^{*}$ is symmetric. Then $T_{\omega}^{*}$ admits a selfadjoint extension if and only if the number of roots of $s-i q$ and $s+i q$ in $\mathbb{D}$, counting multiplicities, coincide.

Proof. The operator $T_{\omega}^{*}$ is an adjoint, and hence closed, and by assumption symmetric. Following definition X.2.12 from [2] we define the deficiency subspaces of $T_{\omega}^{*}$ as the spaces

$$
\begin{aligned}
& \mathcal{L}_{+}=\operatorname{Ker}\left(T_{\omega}^{* *}-i\right)=\left(\operatorname{Ran}\left(T_{\omega}^{*}+i\right)\right)^{\perp}, \\
& \mathcal{L}_{-}=\operatorname{Ker}\left(T_{\omega}^{* *}+i\right)=\left(\operatorname{Ran}\left(T_{\omega}^{*}-i\right)\right)^{\perp},
\end{aligned}
$$

and the deficiency indices as the integers $n_{ \pm}=\operatorname{dim} \mathcal{L}_{ \pm}$. Since $T_{\omega}^{* *}=T_{\omega}$, we have

$$
n_{+}=\operatorname{dim} \operatorname{Ker}\left(T_{\omega}-i\right) \text { and } n_{-}=\operatorname{dim} \operatorname{Ker}\left(T_{\omega}+i\right) .
$$

Also, we have $T_{\omega} \pm i=T_{\omega \pm i}$. By item (b) of Theorem X.2.20 in [2], $T_{\omega}$ has a selfadjoint extension if and only if $n_{+}=n_{-}$. Note that $\omega \pm i=(s \pm i q) / q$. We now apply Corollary 4.2 from [4] to $T_{\omega \pm i}$, to obtain that $n_{ \pm}$is equal to the maximum of 0 and the difference of $m$ and the number of roots of $s \pm i q$ in $\overline{\mathbb{D}}$, counting multiplicities. However, since $T_{\omega}^{*}$ is symmetric, $\omega$ is proper so the number of roots cannot exceed $m$. Note also that $\omega(\mathbb{T}) \subset \mathbb{R}$, so $s \pm i q$ cannot have roots on $\mathbb{T}$. It thus follows that $T_{\omega}^{*}$ has a selfadjoint extension if and only if the number of roots in $\mathbb{D}$ of $s-i q$ and $s+i q$, counting multiplicities, coincide, as claimed. 
Since $T_{\omega}^{*}$ is never selfadjoint for $\omega \in \operatorname{Rat}(\mathbb{T})$ having at least one pole on $\mathbb{T}$, the formulas for $n_{ \pm}$in the above proof along with item (a) of Theorem X.2.20 in [2] directly give the following corollary.

Corollary 5.5. Let $\omega=s / q \in \operatorname{Rat}(\mathbb{T})$, with $s, q \in \mathcal{P}$ coprime, be such that $T_{\omega}^{*}$ is symmetric. Then $s+i q$ or $s-i q$ must have a root in $\mathbb{D}$.

Proposition 5.4 can be rephrased in terms of the index of the operators $T_{\omega \pm i}$.

Proposition 5.6. Let $\omega=s / q \in \operatorname{Rat}(\mathbb{T})$, with $s, q \in \mathcal{P}$ coprime, be such that $T_{\omega}^{*}$ is symmetric. Then $T_{\omega+i}$ and $T_{\omega-i}$ are both Fredholm and $T_{\omega}^{*}$ admits a selfadjoint extension if and only if the Fredholm indices of $T_{\omega+i}$ and $T_{\omega-i}$ coincide.

Proof. This follows directly from Proposition 5.4 and Theorem 1.1 of [4] applied to $\omega+i$ and $\omega-i$, using that $\omega \pm i=(s \pm i q) / q$.

Corollary 5.7. Let $\omega=s / q \in \operatorname{Rat}(\mathbb{T})$, with $s, q \in \mathcal{P}$ coprime, be such that $T_{\omega}^{*}$ is symmetric. Assume $\omega(\mathbb{T}) \neq \mathbb{R}$. Then $T_{\omega}^{*}$ admits a selfadjoint extension.

Proof. The Fredholm index of $T_{\omega-\lambda}$ is constant with respect to $\lambda \in \mathbb{C}$ on the connected components of $\mathbb{C}$ separated by the essential spectrum of $T_{\omega}$, which is equal to $\omega(\mathbb{T})$; see $[5$, Theorem 1.1]. Hence if $\omega(\mathbb{T}) \neq \mathbb{R}$, but $\omega(\mathbb{T}) \subset \mathbb{R}$ since $T_{\omega}^{*}$ is symmetric, then $i$ and $-i$ are in the same connected component and thus $T_{\omega+i}$ and $T_{\omega-i}$ have the same index. The conclusion now follows from Proposition 5.6.

Example 5.8. We return to the functions $\omega_{k}(z)=\left(-i \frac{z+1}{z-1}\right)^{k}$ considered in Example 5.3. Since $\omega_{k}(\mathbb{T})=\mathbb{R}_{+}$for $k$ even, we obtain directly from Corollary 5.7 that $T_{\omega_{k}}^{*}$ admits a selfadjoint extension in case $k$ is even.

For odd values of $k$ we have $\omega_{k}(\mathbb{T})=\mathbb{R}$, and thus no conclusion can be drawn from Corollary 5.7. To deal with the odd case we resort to Proposition 5.4. Take $s(z)=(-i)^{k}(z+1)^{k}$ and $q=(z-1)^{k}$ and write $k$ as $k=2 l+1$. The polynomials $s \pm i q$ are given by

$$
\begin{aligned}
s(z) \pm i q(z) & =i\left((-1)^{l+1}(z+1)^{2 l+1} \pm(z-1)^{2 l+1}\right) \\
& =i\left((-1)^{l+1} \sum_{j=0}^{2 l+1}\left(\begin{array}{c}
2 l+1 \\
j
\end{array}\right) z^{j} \pm \sum_{j=0}^{2 l+1}\left(\begin{array}{c}
2 l+1 \\
j
\end{array}\right) z^{j}(-1)^{2 l+1-j}\right) \\
& =i \sum_{j=0}^{2 l+1}\left(\begin{array}{c}
2 l+1 \\
j
\end{array}\right) z^{j}\left((-1)^{l+1} \pm(-1)^{2 l+1-j}\right) \\
& =i \sum_{j=0}^{2 l+1}\left(\begin{array}{c}
2 l+1 \\
j
\end{array}\right) z^{j}\left((-1)^{l+1} \pm(-1)^{j-1}\right) .
\end{aligned}
$$


For odd values of $l$ one obtains:

$$
\begin{aligned}
s(z)-i q(z) & =-2 i\left(\left(\begin{array}{c}
2 l+1 \\
0
\end{array}\right)+\cdots+\left(\begin{array}{c}
2 l+1 \\
2 l-2
\end{array}\right) z^{2 l-2}+\left(\begin{array}{c}
2 l+1 \\
2 l
\end{array}\right) z^{2 l}\right) \\
s(z)+i q(z) & =2 i\left(\left(\begin{array}{c}
2 l+1 \\
1
\end{array}\right) z+\cdots+\left(\begin{array}{c}
2 l+1 \\
2 l-1
\end{array}\right) z^{2 l-1}+\left(\begin{array}{c}
2 l+1 \\
2 l+1
\end{array}\right) z^{2 l+1}\right) \\
& =2 i z\left(\left(\begin{array}{c}
2 l+1 \\
2 l
\end{array}\right)+\cdots+\left(\begin{array}{c}
2 l+1 \\
2
\end{array}\right) z^{2-2}+\left(\begin{array}{c}
2 l+1 \\
0
\end{array}\right) z^{2 l}\right)
\end{aligned}
$$

Observe that $s+i q$ is of the form $i z p_{+}\left(z^{2}\right)$ where $p_{+}$is a real polynomial of degree $2 l$ and that $s-i g$ is of the form $i p_{-}\left(z^{2}\right)$ where $p_{-}$is a real polynomial of degree $2 l$. Because $p_{+}$and $p_{-}$are real polynomials and the fact that $z^{2}$ is the variable rather than $z$ itself, the nonzero roots of $z p_{+}\left(z^{2}\right)$ come either in pairs $(z$ and $-z)$ for real nonzero roots or in quadruples $(z, \bar{z},-z,-\bar{z})$ for nonreal roots, while zero appears as a simple root. Similarly, the roots of $p_{-}\left(z^{2}\right)$ come in pairs $(z$ and $-z)$ or quadruples $(z, \bar{z},-z,-\bar{z})$ and there is no root at zero. Hence $s+i q$ has an odd number of roots inside the unit disc, and $s-i q$ has an even number of roots inside the unit disc, so that the indices $n_{+}$and $n_{-}$can never coincide. One further observes that $p_{-}=p_{+}^{\sharp}$. In a similar way, for even values of $l$ the polynomial $s+i q$ will have an even number of roots inside the unit disc and $s-i q$ will have an odd number of roots inside the unit disc. Hence, in all cases where $k$ is odd, $T_{\omega}^{*}$ does not have a selfadjoint extension.

We now present a proposition that rephrases the criteria of Proposition 5.4 in terms of the roots of $s+i q$ ( or $s-i q$ ) only. The observation that $T_{\omega_{k}}^{*}$ in Example 5.8 has no selfadjoint extension follows as a special case. In general, $T_{\omega}^{*}$ cannot have a selfadjoint extension whenever $\operatorname{deg}(q)$ is odd for any $\omega \in \operatorname{Rat}(\mathbb{T})$.

Proposition 5.9. Let $\omega=s / q \in \operatorname{Rat}(\mathbb{T})$, with $s, q \in \mathcal{P}$ coprime, be such that $T_{\omega}^{*}$ is symmetric. Set $l_{ \pm}=m-\operatorname{deg}(s \pm i q)$ and define $k_{ \pm, 1}=\#\left\{\begin{array}{l}\text { zeroes of } \omega \pm i \text { inside } \mathbb{T} \\ \text { multi. taken into account }\end{array}\right\}, k_{ \pm, 2}=\#\left\{\begin{array}{l}\text { Eeroes of } \omega \pm i \text { outside } \mathbb{T} \\ \text { multi. taken into account }\end{array}\right\}$. Then

$T_{\omega}^{*}$ has a selfadjoint extension $\Leftrightarrow l_{+}+k_{+, 2}=k_{+, 1} \Leftrightarrow l_{-}+k_{-, 2}=k_{-, 1}$. In particular, if $T_{\omega}^{*}$ has a selfadjoint extension, then $\operatorname{deg}(q)$ must be even.

The basis for the proof of Proposition 5.9 lies in the following lemma, which clarifies the relation between $s+i q$ and $s-i q$ under the assumption that $T_{\omega}^{*}$ is symmetric.

Lemma 5.10. Let $\omega=s / q \in \operatorname{Rat}(\mathbb{T})$, with $s, q \in \mathcal{P}$ coprime, be such that $T_{\omega}^{*}$ is symmetric. Set $l_{ \pm}=\operatorname{deg}(q)-\operatorname{deg}(s \pm i q)$ and let $\gamma$ be the unimodular constant such that $q=\gamma q^{\sharp}$. Then

$$
s \pm i q=\gamma z^{l} \mp(s \mp i q)^{\sharp} .
$$

Moreover, we have $l_{ \pm}=0$ if and only if $\omega(0)= \pm i$. In particular, only one of $l_{+}$and $l_{-}$can be nonzero. 
Proof. Since $T_{\omega}^{*}$ is symmetric, by assumption, $\omega$ has the properties listed in Theorem 5.1. In particular, $\omega$ is proper, $m:=\operatorname{deg}(q) \geq \operatorname{deg}(s)=: n$, and $s=z^{m-n} \widetilde{s}$ with $\widetilde{s}$ self-inversive and the unimodular constants that establish the self-inversiveness of $\widetilde{s}$ and $q$ coincide (equivalently, $q_{0} \overline{s_{n}}=\overline{q_{m}} s_{m-n}$ ).

Note that $\operatorname{deg}(s \pm i q) \neq m$ occurs precisely when $\operatorname{deg}(s)=\operatorname{deg}(q)$ and the leading coefficients $s_{m}$ and $q_{m}$ of $s$ and $q$, respectively, satisfy $s_{m} \pm i q_{m}=0$, i.e., $s_{m} / q_{m}=\mp i$. Since $m=n$, the identity $q_{0} \overline{s_{n}}=\overline{q_{m}} s_{m-n}$ shows $\omega(0)=$ $s_{0} / q_{0}=\overline{s_{m}} / \overline{q_{m}}$. Hence $\operatorname{deg}(s \pm i q) \neq m$ holds if and only if $\omega(0)=\overline{\mp i}= \pm i$, as claimed.

We first prove $(5.1)$ for the case $\omega(0)=0$. So assume $\omega(0)=0$, or equivalently, $s(0)=0$. In this case $l_{+}=l_{-}=0$. Since $s=z^{m-n} \widetilde{s}$ and $\widetilde{s}(0) \neq 0$ (because $\widetilde{s}$ is self-inversive), we have $m>n$. Also note that $m-n$ is equal to the multiplicity of 0 as a root of $s$. We now employ Lemma 2.2, using that $\operatorname{deg}(s+i q)=m=\operatorname{deg}(i q)$, to obtain

$$
\begin{aligned}
\gamma(s \mp i q)^{\sharp} & =z^{\operatorname{deg}(s+i q)-\operatorname{deg}(s)} \gamma s^{\sharp} \mp(-i) \gamma q^{\sharp}=z^{m-n} \gamma \widetilde{s}^{\sharp} \pm i q \\
& =z^{m-n} \widetilde{s} \pm i q=s \pm i q .
\end{aligned}
$$

Hence (5.1) holds.

Now assume $\omega(0) \neq 0$, i.e., $s(0) \neq 0$. In that case $s=\widetilde{s}$. Hence $s$ is self-inversive with the same constant $\gamma$ that establishes the self-inversiveness of $q$. This also yields $m=n$. Since $s$ and $q$ are self-inversive with the same constant $\gamma$, we have

$$
\overline{s_{m-k}} q_{k}=\overline{q_{m-k} s_{m-k}} \gamma=\overline{q_{m-k}} s_{k} \quad \text { for } k=0, \ldots, m .
$$

Hence for all $k$ we have

$$
\begin{aligned}
\overline{s_{m-k}}\left(s_{k}+i q_{k}\right) & =s_{k}\left(\overline{s_{m-k}}+i \overline{q_{m-k}}\right) \text { and } \overline{q_{m-k}}\left(s_{k}+i q_{k}\right) \\
& =q_{k}\left(\overline{s_{m-k}}+i \overline{q_{m-k}}\right) .
\end{aligned}
$$

In case $s_{m-k}=0$ and $q_{m-k}=0$, also $s_{k}=0$ and $q_{k}=0$, since $s_{k}=\gamma \overline{s_{m-k}}$ and $q_{k}=\gamma \overline{q_{m-k}}$, and thus $s_{k}+i q_{k}=0=\gamma\left(\overline{s_{m-k}}+i \overline{q_{m-k}}\right)$. If either $s_{m-k} \neq 0$ or $q_{m-k} \neq 0$, divide the first identity by $\overline{s_{m-k}}$ or the second identity by $\overline{q_{m-k}}$ to arrive at $s_{k}+i q_{k}=\gamma\left(\overline{s_{m-k}}+i \overline{q_{m-k}}\right)$. Hence

$$
s_{k}+i q_{k}=\gamma\left(\overline{s_{m-k}-i q_{m-k}}\right) \quad \text { for } k=0, \ldots, m \text {. }
$$

Thus $s_{k}+i q_{k}=0$ if and only if $s_{m-k}-i q_{m-k}=0$. It follows that 0 is a root of $s \pm i q$ with multiplicity $l_{\mp}$. Comparing coefficients, it follows that the identities in (5.1) correspond to the identities in (5.2). Hence (5.1) holds.

Proof of Proposition 5.9. Since $T_{\omega}^{*}$ is assumed to be symmetric, (5.1) holds. Together with the fact that the $\sharp$ operator reflects roots over $\mathbb{T}$, this implies that the number of roots of $s \pm i q$ inside $\mathbb{T}$ are equal to $l_{ \pm}$plus the number of roots of $s \mp i q$ outside $\mathbb{T}$, counting multiplicities. In other words, we have

$$
k_{+, 1}=l_{-}+k_{-, 2} \quad \text { and } \quad k_{-, 1}=l_{+}+k_{+, 2} .
$$

By Proposition 5.6, $T_{\omega}^{*}$ has a selfadjoint extension if and only if $s+i q$ and $s-i q$ have an equal number of roots inside $\mathbb{T}$, again counting multiplicities, equivalently, $k_{+, 1}=k_{-, 1}$. Given (5.3), it follows that $k_{+, 1}=k_{-, 1}$ is equivalent 
to $k_{+, 1}=l_{+}+k_{+, 2}$, and likewise to $k_{-, 1}=l_{-}+k_{-, 2}$. This proves the two criteria for $T_{\omega}^{*}$ to have a selfadjoint extension.

By Lemma 5.10, either $l_{+}=0$ or $l_{-}=0$. Say $l_{+}=0$. Since $s+i q$ cannot have roots on $\mathbb{T}$, we have $\operatorname{deg}(q)=\operatorname{deg}(s+i q)=k_{+, 1}+k_{+, 2}$. If $T_{\omega}^{*}$ admits a selfadjoint extension, then we have $k_{+, 1}=l_{+}+k_{+, 2}=k_{+, 2}$. Hence $\operatorname{deg}(q)=2 k_{+, 1}$ is even. For $l_{-}=0$ the arguments goes similarly.

Combining the fact that $T_{\omega}^{*}$ cannot have a selfadjoint extension in case $\omega=s / q \in \operatorname{Rat}(\mathbb{T}), s, q$ co-prime, and $\operatorname{deg}(q)$ odd with Corollary 5.7 immediately yields the following result.

Corollary 5.11. Let $\omega=s / q \in \operatorname{Rat}(\mathbb{T})$, with $s, q \in \mathcal{P}$ co-prime, be such that $T_{\omega}^{*}$ is symmetric and $\operatorname{deg}(q)$ is odd. Then $\omega(\mathbb{T})=\mathbb{R}$.

The next example shows that also with $\operatorname{deg}(q)$ even it can occur that $T_{\omega}^{*}$ does not admit a selfadjoint extension.

Example 5.12. Let $\omega=s / q$ with

$$
s(z)=i\left(1+a z+z^{2}\right), \text { for some } 0 \neq a \in \mathbb{R}, \quad \text { and } \quad q(z)=1-z^{2} .
$$

Then $m=n$ and

$$
s^{\sharp}=-s, \quad q^{\sharp}=-q .
$$

So $T_{\omega}^{*}$ is symmetric by Theorem 5.1 (5). Also, we have

$$
(s+i q)(z)=i(2+a z) \text { and }(s-i q)(z)=i z(a+2 z) .
$$

Hence the number of roots of $s-i q$ inside $\mathbb{D}$ is 1 if $|a| \geq 2$ and 2 if $0 \neq|a|<2$, while the number of roots of $s+i q$ inside $\mathbb{D}$ is 1 if $|a|>2$ and 0 if $0 \neq|a| \leq 2$. Thus $T_{\omega}^{*}$ admits a selfadjoint extension if and only if $|a|>2$.

\section{Comparison with the Unbounded Toeplitz Operator Defined by Sarason}

The Smirnov class $N^{+}$consists of quotients $\frac{b}{a}$ with $a$ and $b H^{\infty}$-functions such that the denominator $a$ is an outer function. The function $\varphi=\frac{b}{a} \in N^{+}$ is said to be in canonical form if $a(0)>0$ and $|a|^{2}+|b|^{2}=1$ on $\mathbb{T}$. By Proposition 3.1 of [11], every function $\varphi \in N^{+}$can be uniquely written in canonical form.

In [11], Sarason investigated an unbounded Toeplitz operator $T_{\varphi}^{\mathrm{Sa}}$ with symbol $\varphi$ in $N^{+}$, which is defined by

$$
\operatorname{Dom}\left(T_{\varphi}^{\mathrm{Sa}}\right)=\left\{f \in H^{2}: \varphi f \in H^{2}\right\}, \quad T_{\varphi}^{\mathrm{Sa}} f=\varphi f \quad\left(f \in \operatorname{Dom}\left(T_{\varphi}^{S a}\right)\right) .
$$

More generally, $T_{\varphi}^{\mathrm{Sa}}$ can be defined in this way for any holomorphic function $\varphi$ on $\mathbb{D}$, but for $T_{\varphi}^{\mathrm{Sa}}$ to be densely defined, $\varphi$ must be in $N^{+}[11$, Lemma 5.2].

Let $\varphi=\frac{b}{a} \in N^{+}$be the canonical representation of $\varphi$. Then it is shown in Proposition 5.3 of [11] that $\operatorname{Dom}\left(T_{\varphi}^{\mathrm{Sa}}\right)=a H^{2}$. The adjoint of the operator $T_{\varphi}^{\mathrm{Sa}}$ is motivated by the action of the conjugate transpose of the matrix representation of $T_{\varphi}^{\mathrm{Sa}}$, which is lower triangular. The domain of the adjoint 
operator is shown to contain the space $H(\overline{\mathbb{D}})$ of functions that are analytic on some neighborhood of the closed unit disc $\overline{\mathbb{D}}$, and the adjoint is equal to the closure of the operator on $H(\overline{\mathbb{D}})$; see [11, Lemmas 6.1 and 6.4].

Let $\omega=s / q \in \operatorname{Rat}(\mathbb{T})$ with $s, q \in \mathcal{P}$ co-prime. Set $n=\operatorname{deg}(s)$ and $m=$ $\operatorname{deg}(q)$. Assume $\omega$ is proper, i.e., $n \leq m$. Then $\omega^{*}(z)=z^{m-n} s^{\sharp} / q^{\sharp} \in \operatorname{Rat}(\mathbb{T})$. Since $q^{\sharp}$ has zeroes only on $\mathbb{T}$ it is outer and thus $\omega^{*} \in N^{+}$. While in general $T_{\omega}$ and $T_{\omega}^{\mathrm{Sa}}$ are different, the following proposition shows that $T_{\omega}$ coincides with $T_{\omega^{*}}^{\mathrm{Sa}}$, and hence $T_{\omega}=T_{\omega}^{* *}=T_{\omega^{*}}^{\mathrm{Sa}}$. Without the properness assumption, $\omega^{*}$ is not in $N^{+}$, because $\omega^{*}$ has a pole at 0 , and hence $T_{\omega^{*}}^{\mathrm{Sa}}$ is not defined.

Proposition 6.1. Let $\widetilde{\omega}=\widetilde{s} / \widetilde{q} \in \operatorname{Rat}(\mathbb{T})$ with $\widetilde{s}, \widetilde{q} \in \mathcal{P}$ co-prime. Then Dom $\left(T_{\widetilde{\omega}}^{S a}\right)=\widetilde{q} H^{2}$ and $T_{\widetilde{\omega}}^{S a}=\left.T_{\widetilde{\omega}}\right|_{\widetilde{q} H^{2}}$. In particular, if $\omega \in \operatorname{Rat}(\mathbb{T})$ is proper, then $T_{\omega}^{*}=T_{\omega^{*}}^{S a}$.

Proof. We first show $\operatorname{Dom}\left(T_{\widetilde{\omega}}^{\mathrm{Sa}}\right)=\widetilde{q} H^{2}$. Let $\widetilde{\omega}=a / b$ be the canonical form of $\widetilde{\omega}$. As noted above, $\operatorname{Dom}\left(T_{\widetilde{\omega}}^{\mathrm{Sa}}\right)=a H^{2}$. By the Fejér-Riesz Theorem there is a polynomial $r$ such that on $\mathbb{T}$ we have $|r|^{2}=|\widetilde{s}|^{2}+|\widetilde{q}|^{2}, r$ has no roots in $\mathbb{D}$ and $\arg (r(0))=\arg (\widetilde{q}(0))$. The latter is possible since $\widetilde{q}(0) \neq 0$ and implies $\widetilde{q}(0) / r(0)>0$. Note that $r$ also has no roots on $\mathbb{T}$, since $\widetilde{s}$ and $\widetilde{q}$ are co-prime. It follows that $\widetilde{q} / r$ and $\widetilde{s} / r$ are both $H^{\infty}$-functions, $\widetilde{q} / r$ is outer and $\widetilde{q}(0) / r(0)>0$. Hence $a=\widetilde{q} / r$ and $b=\widetilde{s} / r$, by the uniqueness of the canonical form. Also, since all the roots of $r$ are outside $\mathbb{T}, r^{-1} H^{2}=H^{2}$, so that $a H^{2}=\widetilde{q} H^{2}$.

Now let $f \in \operatorname{Dom}\left(T_{\widetilde{\omega}}^{\mathrm{Sa}}\right)$, say $f=\widetilde{q} h$ with $h \in H^{2}$. Then $T_{\widetilde{\omega}}^{\mathrm{Sa}} f=\widetilde{\omega} f=\widetilde{s} h$. On the other hand, the fact that $\widetilde{\omega} f=\widetilde{s} h$ and $\widetilde{s} h \in H^{2}$ shows $T_{\widetilde{\omega}} f=\mathbb{P} \widetilde{s} h=$ $\widetilde{s}$. Hence $T_{\widetilde{\omega}}^{\mathrm{Sa}}=\left.T_{\widetilde{\omega}}\right|_{\widetilde{q} H^{2}}$.

Next we employ some of the ideas from [11] to derive the following result. Recall that for a Hilbert space operator $T: \operatorname{Dom}(T) \rightarrow \mathcal{H}$ a linear submanifold $\mathcal{D} \subset \operatorname{Dom}(T)$ is called a core in case the graph $G\left(\left.T\right|_{\mathcal{D}}\right)$ of $\left.T\right|_{\mathcal{D}}$ is dense in the graph $G(T)$ of $T$; cf., page 166 in [7].

Theorem 6.2. Let $\omega \in \operatorname{Rat}(\mathbb{T})$. Then $H(\overline{\mathbb{D}})$ is contained in $\operatorname{Dom}\left(T_{\omega}\right)$. If $\omega$ is proper, then $H(\overline{\mathbb{D}})$ is a core of $T_{\omega}$.

Proof of $H(\overline{\mathbb{D}}) \subset \operatorname{Dom}\left(T_{\omega}\right)$. Write $\omega=\frac{s}{q} \in \operatorname{Rat}_{0}(\mathbb{T})$ with $s, q \in \mathcal{P}$ coprime. Let $f \in H(\overline{\mathbb{D}})$. Then there exists a $R>1$ such that $f$ is still analytic on an open neighborhood of the closed disc with radius $R$. Set $\tilde{f}(z)=f(R z)$, $\widetilde{q}(z)=q(R z)$ and $\widetilde{s}(z)=s(R z)$. Then $\widetilde{f} \in H^{2}$ and $\widetilde{q}$ is a polynomial with no roots on $\mathbb{T}$ and $\operatorname{deg}(q)=\operatorname{deg}(\widetilde{q})$. By Theorem 3.1 in [4], $H^{2}=\widetilde{q} H^{2}+\mathcal{P}_{\operatorname{deg}(q)-1}$. Thus $\widetilde{s} \widetilde{f}=\widetilde{q} \tilde{h}+\widetilde{r}$ for some $\widetilde{h} \in H^{2}$ and $\widetilde{r} \in \mathcal{P}$ with $\operatorname{deg}(\widetilde{r})<\operatorname{deg}(q)$. Now set $r(z)=\widetilde{r}(z / R)$ and $h(z)=\widetilde{h}(z / R)$. Then $r \in \mathcal{P}$ with $\operatorname{deg}(r)=\operatorname{deg}(\widetilde{r})<$ $\operatorname{deg}(q)$ and $h \in H^{2}$, even $h \in H(\overline{\mathbb{D}})$. Also, we have $s f=q h+r$. Thus $f \in \operatorname{Dom}\left(T_{\omega}\right)$.

Before proving the second claim of Theorem 6.2 it is useful to consider the value of $T_{\omega}$ when applied to the evaluation functional or reproducing kernel element $k_{\lambda}(z)=(1-\bar{\lambda} z)^{-1}$, where $\lambda \in \mathbb{D}$. Note that $k_{\lambda} \in H(\overline{\mathbb{D}})$, hence $k_{\lambda} \in H^{2}$, and $k_{\lambda}$ has the reproducing kernel property for $H^{2}$ :

$\operatorname{span}\left\{k_{\lambda}: \lambda \in \mathbb{D}\right\}$ dense in $H^{2} \quad$ and $\quad\left\langle h, k_{\lambda}\right\rangle=h(\lambda) \quad\left(h \in H^{2}, \lambda \in \mathbb{D}\right)$. 
See [8] for a recent account of the theory of reproducing kernel Hilbert spaces and further references.

Lemma 6.3. Let $\omega=s / q \in \operatorname{Rat}(\mathbb{T})$, with $s, q \in \mathcal{P}$ co-prime, be proper. Then

$$
T_{\omega} k_{\lambda}=\overline{\omega^{*}(\lambda)} k_{\lambda} \quad(\lambda \in \mathbb{D}) .
$$

Proof. Suppose $g=T_{\omega} k_{\lambda}$ then $s(z)(1-\bar{\lambda} z)^{-1}=q(z) g(z)+r(z)$, where $r \in \mathcal{P}_{m-1}$. Here $m=\operatorname{deg}(q)$. Hence $(1-\bar{\lambda} z) g=(s+(1-\bar{\lambda} z) r) / q$ is in $\operatorname{Rat}(\mathbb{T})$ as well as in $H^{2}$. This can only occur if $(1-\bar{\lambda} z) g$ is a polynomial, i.e., $g=k_{\lambda} \widetilde{r}$ for some $\widetilde{r} \in \mathcal{P}$. Thus $s+(1-\bar{\lambda} z) r=q \widetilde{r}$. Since $\omega$ is proper, the degree of the left hand side is at most $m$. But then $\widetilde{r}$ is constant, say with value $\widetilde{c}$. This shows $T_{\omega} k_{\lambda}=\widetilde{c} k_{\lambda}$.

To determine $\widetilde{c}$ we evaluate the identity $s+(1-\bar{\lambda} z) r=q \widetilde{c}$ at $1 / \bar{\lambda}$. This gives $s(1 / \bar{\lambda})=q(1 / \bar{\lambda}) \widetilde{c}$. Note that

$$
s^{\sharp}(\lambda)=\lambda^{n} \overline{s(1 / \bar{\lambda})} \text { and } q^{\sharp}(\lambda)=\lambda^{m} \overline{q(1 / \bar{\lambda})},
$$

where $n=\operatorname{deg}(s)$. Hence

$$
s(1 / \bar{\lambda})=\bar{\lambda}^{-n \overline{s^{\sharp}(\lambda)}} \text { and } \quad q(1 / \bar{\lambda})=\bar{\lambda}^{-m} \overline{q^{\sharp}(\lambda)} .
$$

This gives

$$
\widetilde{c}=\frac{\bar{\lambda}^{-n} \overline{s^{\sharp}(\lambda)}}{\bar{\lambda}^{-m} \overline{q^{\sharp}(\lambda)}}=\overline{\left(\frac{\lambda^{m-n} s^{\sharp}(\lambda)}{q^{\sharp}(\lambda)}\right)}=\overline{\omega^{*}(\lambda)} .
$$

Proof of Theorem 6.2. It remains to prove that $H(\overline{\mathbb{D}})$ is a core for $T_{\omega}$ in case $\omega$ is proper. So, assume $\omega$ is proper. We need to show that the graph of $\left.T_{\omega}\right|_{H(\overline{\mathbb{D}})}$ is dense in the graph of $T_{\omega}$. In other words, let $f, g \in H^{2}$ with $(f, g)$ perpendicular to $G\left(\left.T_{\omega}\right|_{H(\overline{\mathbb{D}})}\right)$, then we need to show $(f, g)$ is perpendicular to $G\left(T_{\omega}\right)$. Since $k_{\lambda} \in H(\overline{\mathbb{D}})$, for $\lambda \in \mathbb{D}$, we have

$0=\left\langle(f, g),\left(k_{\lambda}, T_{\omega} k_{\lambda}\right)\right\rangle=\left\langle f, k_{\lambda}\right\rangle+\left\langle g, \overline{\omega^{*}(\lambda)} k_{\lambda}\right\rangle=f(\lambda)+\omega^{*}(\lambda) g(\lambda)(\lambda \in \mathbb{D})$.

Hence $\omega^{*} g=-f$. In particular, $\omega^{*} g \in H^{2}$. Thus $g \in \operatorname{Dom}\left(T_{\omega^{*}}^{\mathrm{Sa}}\right)=\operatorname{Dom}\left(T_{\omega}^{*}\right)$ and $T_{\omega}^{*} g=-f$, by Proposition 6.1. For any $h \in \operatorname{Dom}\left(T_{\omega}\right)$ we have

$$
\left\langle(f, g),\left(h, T_{\omega} h\right)\right\rangle=\left\langle\left(-T_{\omega}^{*} g, g\right),\left(h, T_{\omega} h\right)\right\rangle=-\left\langle T_{\omega}^{*} g, h\right\rangle+\left\langle g, T_{\omega} h\right\rangle=0 .
$$

In Section 8 of [11], Sarason introduced the class of closed, densely defined operators $T$ on $H^{2}$ which satisfy

(1) $T_{z} \operatorname{Dom}(T) \subset \operatorname{Dom}(T)$;

(2) $T_{z}^{*} T T_{z}=T$

(3) $f \in \operatorname{Dom}(T), f(0)=0 \Rightarrow T_{z}^{*} f \in \operatorname{Dom}(T)$.

This class of operators was further studied by Rosenfeld in $[9,10]$ in which he referred to such operators as Sarason-Toeplitz operators. The operators $T_{\varphi}^{\mathrm{Sa}}$, for $\varphi \in N^{+}$, are Sarason-Toeplitz operators, and the class of operators 
is closed under taking adjoints, by Proposition 2.1 in [10]. Hence, by Proposition $6.1, T_{\omega}$ is a Sarason-Toeplitz operator whenever $\omega \in \operatorname{Rat}(\mathbb{T})$ is proper. We show that in fact $T_{\omega}$ is a Sarason-Toeplitz operator for any $\omega \in$ Rat.

Proposition 6.4. Let $\omega \in$ Rat. Then $T_{\omega}$ on $H^{2}$ is a Sarason-Toeplitz operator.

Proof. First consider $\omega \in \operatorname{Rat}(\mathbb{T})$. That $T_{\omega}$ satisfies (1) and (2) was proved in $\left[4\right.$, Lemma 2.3]. We claim that $T_{z}^{*} \operatorname{Dom}\left(T_{\omega}\right) \subset \operatorname{Dom}\left(T_{\omega}\right)$. Write $\omega=s / q$ with $s, q \in \mathcal{P}$ co-prime. Then $\operatorname{Dom}\left(T_{\omega}\right)=q H^{2}+\mathcal{P}_{\operatorname{deg}(q)-1}$. Let $f=q h+$ $r \in \operatorname{Dom}\left(T_{\omega}\right)$ with $h \in H^{2}$ and $r \in \mathcal{P}, \operatorname{deg}(r)<\operatorname{deg}(q)$. Then $T_{z}^{*} f=$ $q T_{z}^{*} h+h(0) T_{z}^{*} q+T_{z}^{*} r$, which is in $q H^{2}+\mathcal{P}_{\operatorname{deg}(q)-1}=\operatorname{Dom}\left(T_{\omega}\right)$. Hence $T_{\omega}$ is a Sarason-Toeplitz operator in case $\omega \in \operatorname{Rat}(\mathbb{T})$.

Now take $\omega \in$ Rat arbitrarily. By Lemma 5.1 in [4], see also Sect. 4 above, $\omega=\omega_{-} z^{\kappa} \omega_{0} \omega_{+}$with $\kappa \in \mathbb{Z}$, and $\omega_{-}, \omega_{0}$ and $\omega_{+}$in Rat with zeroes and poles only inside, on or outside $\mathbb{T}$, respectively. In particular, $\omega_{0} \in \operatorname{Rat}(\mathbb{T})$, $\omega_{-}$and $\omega_{-}^{-1}$ are both anti-analytic, and $\omega_{+}$and $\omega_{+}^{-1}$ are both analytic. Also, $T_{\omega}=T_{\omega_{-}} T_{z^{\kappa} \omega_{0}} T_{\omega_{+}}$. Note that $z^{\kappa} \omega_{0} \in \operatorname{Rat}(\mathbb{T})$ in case $\kappa \geq 0$ and $T_{z^{\kappa} \omega_{0}}=$ $T_{z^{\kappa}} T_{\omega_{0}}$ in case $\kappa<0$ (by [4, Lemma 5.3]). In both cases it now easily follows that $T_{z^{\kappa} \omega_{0}}$ is a Sarason-Toeplitz operator. The claim for $T_{\omega}$ follows since $T_{\omega_{+}}^{ \pm 1} T_{z}=T_{z} T_{\omega_{+}}^{ \pm 1}$ and $T_{\omega_{-}}^{ \pm 1} T_{z}^{*}=T_{z}^{*} T_{\omega_{-}}^{ \pm 1}$.

In fact, by the same arguments one can show that $T_{\omega}$ on $H^{p}, 1<p<\infty$, satisfies (1)-(3) in case $T_{z}^{*}$ is replaced by $T_{z^{-1}}$.

\section{Acknowledgements}

This work is based on research supported in part by the National Research Foundation of South Africa. Any opinion, finding and conclusion or recommendation expressed in this material is that of the authors and the NRF does not accept any liability in this regard.

Open Access. This article is distributed under the terms of the Creative Commons Attribution 4.0 International License (http://creativecommons.org/licenses/by/4. $0 /$ ), which permits unrestricted use, distribution, and reproduction in any medium, provided you give appropriate credit to the original author(s) and the source, provide a link to the Creative Commons license, and indicate if changes were made.

Publisher's Note Springer Nature remains neutral with regard to jurisdictional claims in published maps and institutional affiliations.

\section{References}

[1] Cohn, A.: Über die Anzahl der Wurzeln einer algebraischen Gleichung in einem Kreise. Math. Z. 14, 110-148 (1922)

[2] Conway, J.B.: A Course in Functional Analysis. Graduate Texts in Mathematics, vol. 96, 2nd edn. Springer, New York (1990)

[3] Goldberg, S.: Unbounded Linear Operators: Theory and Applications. McGraw-Hill, New York (1966)

[4] Groenewald, G.J., ter Horst, S., Jaftha, J., Ran, A.C.M.: A Toeplitz-like operator with rational symbol having poles on the unit circle I: Fredholm properties. Oper. Theory Adv. Appl. 271, 239-268 (2018) 
[5] Groenewald, G.J., ter Horst, S., Jaftha, J., Ran, A.C.M.: A Toeplitz-like operator with rational symbol having poles on the unit circle II: the spectrum. Oper. Theory Adv. Appl. 272, 133-154 (2019)

[6] Helson, H.: Large analytic functions. Oper. Theory Adv. Appl. 43, 209-216 (1990)

[7] Kato, T.: Perturbation Theory for Linear Operators. Classics in Mathematics. Springer, Berlin (1995). Reprint of the 1980 edition

[8] Paulsen, V.I., Raghupathi, M.: An Introduction to the Theory of Reproducing kernel Hilbert spaces. Cambridge Studies in Advanced Mathematics, vol. 152. Cambridge University Press, Cambridge (2016)

[9] Rosenfeld, J.A.: Classes of densely defined multiplication and Toeplitz operators with applications to extensions of RKHS's. Ph.D. Thesis, University of Florida (2013)

[10] Rosenfeld, J.A.: The Sarason sub-symbol and the recovery of the symbol of densely defined Toeplitz operators over the Hardy space. J. Math. Anal. Appl. 440, 911-921 (2016)

[11] Sarason, D.: Unbounded Toeplitz operators. Integr. Equ. Oper. Theory 61, 281-298 (2008)

[12] Schechter, M.: The conjugate of a product of operators. J. Funct. Anal. 6, 26-28 (1970)

[13] van Casteren, J.A.W., Goldberg, S.: The conjugate of the products of operators. Stud. Math. 38, 125-130 (1970)

[14] Yosida, K.: Functional Analysis. Fundamental Principles of Mathematical Sciences, vol. 123, 6th edn. Springer, New York (1980)

G. J. Groenewald, S. ter Horst and A. C. M. Ran

Department of Mathematics, Unit for BMI

North-West University

Potchefstroom 2531

South Africa

e-mail: Gilbert.Groenewald@nwu.ac.za

S. ter Horst

e-mail: Sanne.TerHorst@nwu.ac.za

S. ter Horst

DST-NRF Centre of Excellence in Mathematical and Statistical Sciences (CoEMaSS)

Johannesburg

South Africa

J. Jaftha

Numeracy Centre

University of Cape Town

Rondebosch, Cape Town 7701

South Africa

e-mail: Jacob.Jaftha@uct.ac.za 


\author{
A. C. M. Ran $(\bowtie)$ \\ Department of Mathematics, Faculty of Science \\ Vrije Universiteit Amsterdam \\ De Boelelaan 1081a \\ 1081 HV Amsterdam \\ The Netherlands \\ e-mail: a.c.m.ran@vu.nl
}

Received: November 16, 2018.

Revised: August 28, 2019. 